\title{
Vector-boson pair production and electroweak corrections in HERWIG++
}

\author{
Stefan Gieseke ${ }^{1}$, Tobias Kasprzik ${ }^{2, a}$, Johann H. Kühn ${ }^{2}$ \\ ${ }^{1}$ Karlsruhe Institute of Technology (KIT), Institut für Theoretische Physik (IThP), 76131 Karlsruhe, Germany \\ ${ }^{2}$ Karlsruhe Institute of Technology (KIT), Institut für Theoretische Teilchenphysik (TTP), 76131 Karlsruhe, Germany
}

Received: 29 January 2014 / Accepted: 19 July 2014 / Published online: 12 August 2014

(C) The Author(s) 2014. This article is published with open access at Springerlink.com

\begin{abstract}
The detailed study of vector-boson pair production processes at the LHC will lead to a better understanding of electroweak physics. As pointed out before, a consistent inclusion of higher-order electroweak effects in the analysis of corresponding experimental data may be crucial to properly predict the relevant phenomenological features of these important reactions. Those contributions lead to dramatic distortions of invariant-mass and angular distributions at high energies, but may also significantly affect the cross section near threshold, as is the case e.g. for Zpairs. For this reason, we present an analysis of the nextto-leading-order electroweak corrections to $\mathrm{WW}, \mathrm{WZ}$, and $\mathrm{ZZ}$ production at the LHC, taking into account mass effects as well as leptonic decays. Hence, our predictions are valid in the whole kinematic reach of the LHC and, moreover, respect the spin correlations of the leptonic decay products at next-to-leading-order accuracy. Starting from these fixedorder results, a simple and straightforward method is motivated to combine the electroweak corrections with state-ofthe-art Monte Carlo predictions, focusing on a meaningful combination of higher-order electroweak and QCD effects. To illustrate our approach, the electroweak corrections are implemented in the HERWIG++ generator, and their phenomenological effects within a QCD environment are studied explicitly.
\end{abstract}

\section{Introduction}

Vector-boson pair-production processes play a central role in LHC phenomenology. These processes are not only of great importance with respect to background analyses in Standard-Model (SM) Higgs production, they will also provide deeper insight into the structure of the electroweak interaction at highest energies. This is particularly true for the

\footnotetext{
a e-mail: tobias.kasprzik@kit.edu
}

future high-luminosity run of the LHC at a center-of-mass (CM) energy of $13 \mathrm{TeV}$, which will allow for an unprecedented accuracy in the analysis of vector-boson interactions at the $\mathrm{TeV}$ scale. Consequently, theoretical prediction with high accuracy is needed for this important class of processes.

Given the experimental accuracy already achieved by LHC experiments in the 7- and 8-TeV runs [1-6], at least the next-to-leading-order (NLO) QCD corrections are mandatory for a robust prediction of $V$-boson pair-production processes (for selected references see Refs. [7,8]). In addition, important steps toward NNLO predictions for massive vector-boson pair-production processes have been made [911]. (As far as photon-pair and $Z \gamma$ production at the LHC are concerned, the full strong two-loop corrections are known even fully differentially [12-14]). In particular, approximate NNLO results for $\mathrm{W}^{+} \mathrm{Z}$ and $\mathrm{WW}$ production have been provided for high-transverse-momentum observables [15,16], as well as for WW production in the threshold limit [17]. Recently, also the full NNLO corrections to the total Zpair production cross section were computed [18], reducing the remaining theory uncertainties to a level of only $3 \%$.

Having reached this high level of accuracy in the QCD predictions, also electroweak (EW) corrections (and other related electroweak effects) are becoming more and more important, and a lot of activity has taken place also in this field. In particular, the interplay of EW corrections and anomalous couplings has been investigated in Ref. [19]. The corresponding EW corrections have been computed in Ref. [20] in the high-energy limit, including leptonic decays and off-shell effects. Recently, also the full EW corrections to W-pair production, also taking into account mass effects as well as off-shell effects, have been evaluated for the leptonic final state [21]. Leading two-loop effects at high transverse momenta were evaluated in Ref. [22] for W-pairs. A detailed analysis of on-shell $V$-boson pair production 
$\left(V=\mathrm{W}^{ \pm}, \mathrm{Z}\right)$ and $\gamma \gamma$ production including EW corrections has been provided in Refs. [7,8], consistently including all mass effects. Recently, a detailed review of NLO effects in pair production of massive bosons has been provided, emphasizing the importance of photon-induced contributions [23].

Expecting first results for the full NNLO QCD corrections to W-pair production in the near future [24], a natural next step would be the combination of EW and QCD predictions at $\mathcal{O}\left(\alpha_{s} \alpha\right)$ accuracy on a consistent theory basis, as has been partially done for the Drell-Yan process [25-33] already, where important contributions from mixed $\mathrm{EW} \times \mathrm{QCD}$ corrections in the resonance region were found [34]. However, these multiscale two-loop calculations are beyond feasibility at present. Nevertheless, at least a pragmatic prescription of combining QCD predictions with EW corrections is desirable, aiming for a combination of EW precision with standard Monte Carlo (MC) tools.

In this work we extend the above results in two ways. In Sect. 2, in addition to predictions for $\mathrm{W}$-pair production, also a first study of EW corrections to $\mathrm{WZ}$ and $\mathrm{ZZ}$ production is presented, including mass effects as well as leptonic decays, to allow for a realistic event definition in the leptonic decay modes. We point out that our results are not restricted to the high-energy regime but are also valid at moderate energies of $\sim 200 \mathrm{GeV}$, where already corrections of 5-10\% may be observed. Applying these results we propose, in a second step, a straightforward and simple strategy for the implementation of EW corrections in any state-of-the-art MC generator (Sect. 4), relying on $K$-factors for unpolarized two-by-two scattering and the assumption of factorization of EW and QCD effects, as will be motivated in Sect. 3. Our method will be tested against an alternative implementation method especially tailored for the HERWIG++ [35,36] MC generator. In Sect. 5 selected numerical results obtained within the HERWIG++ setup will be discussed.

\section{Electroweak corrections}

As stated in the introduction, the complete evaluation of the combined QCD and electroweak corrections to gaugeboson pair production of order $\alpha \alpha_{s}$ is presently out of reach. As a first step we, therefore, consider a factorized ansatz, where the kinematics of events with additional QCD radiation is mapped to effective two-body collisions, described by effective (squared) partonic energies and energy transfers $\hat{s}$ and $\hat{t}$. We assume that the bulk of the QCD corrections arises from events with soft or collinear emission, where the factorized ansatz is expected to work well. The EW corrections are taken directly from the result of the oneloop calculation, evaluated at the same kinematical point.
This approach is expected to fail for events with large transverse momenta of the gauge-boson pair recoiling against a jet with large transverse momentum. These events, however, are of lesser relevance for the study of gauge-boson dynamics and can be eliminated by suitable cuts, as discussed in Sect. 4. The motivation of this approach will be discussed in Sect. 3, and details of the implementation are given in Sect. 4.

In the present section we concentrate on the electroweak corrections and motivate that indeed the bulk of the electroweak corrections can be collected in a $K$-factor which is given as a function of $\hat{s}$ and $\hat{t}$ only (Sect. 2.1). Photonic corrections (which evidently lead to a more complicated kinematic situation) can be split off such that the corresponding modifications of the electroweak corrections are small. This aspect will be investigated in Sect. 2.2.

A second simplification is introduced by applying a correction factor which does not depend on the helicities of the gauge bosons. In Sect. 2.3 we argue that this approximation still preserves the proper angular distributions and correlations of the $\mathrm{Z}$ and $\mathrm{W}$ decay products and investigate the phenomenological implications of this approximation in detail. Finally, the corrections as derived for on-shell gaugeboson production are applied for the cases where $\mathrm{W}$ or $\mathrm{Z}$ are slightly off mass shell (in the case of $Z$ bosons we also include the amplitude where fermion pairs are produced through the virtual photon). The quality of this approximation at lowest order will also be discussed in Sect. 2.3. First, however, let us briefly recall the most important phenomenological features of various EW effects in on-shell WW, WZ, and ZZ production at the $\mathrm{LHC}$.

\subsection{On-shell gauge-boson pair productions}

Here, we summarize the combination of different electroweak effects in on-shell $V$-pair production which have been discussed in detail in Refs. [7,8,23]. For the numerical implementation, we use the default setup defined in Refs. [7,8].

To be specific, we use the following SM input parameters for the numerical analysis:

$$
\begin{array}{ll}
G_{\mu}=1.16637 \times 10^{-5} \mathrm{GeV}^{-2}, & \\
M_{\mathrm{W}}=80.398 \mathrm{GeV}, & M_{\mathrm{Z}}=91.1876 \mathrm{GeV}, \\
M_{\mathrm{H}}=125 \mathrm{GeV}, & M_{\mathrm{t}}=173.4 \mathrm{GeV} .
\end{array}
$$

For the evaluation of all tree-level contributions we assume a block-diagonal CKM matrix with

$$
\left|V_{\mathrm{ud}}\right|=\left|V_{\mathrm{cs}}\right|=0.974, \quad\left|V_{\mathrm{us}}\right|=\left|V_{\mathrm{cd}}\right|=\sqrt{1-\left|V_{\mathrm{ud}}\right|^{2}} .
$$


Ignoring, furthermore, quark masses within the first two families, both tree-level and one-loop predictions for $\mathrm{ZZ}$ are equivalent to those without quark mixing. As a consequence of the smallness of the bottom-quark PDF the tree-level contribution from $b \bar{b}$ annihilation to $\mathrm{ZZ}$ is small to start with. In addition, the non-diagonal CKM elements involving b quarks are small, and the ansatz (2.2) is well justified. As a consequence, $\mathrm{b} \overline{\mathrm{b}} \rightarrow \mathrm{ZZ}$ can safely be handled within the third family. ${ }^{1}$ The situation is different for the WZ channel. In this case, the interplay between CKM angles and PDFs leads to a shift of the tree-level prediction of about one percent. For the radiative corrections the CKM matrix can, therefore, still be set to unity.

In the on-shell scheme applied in our computation, the weak mixing angle $\cos ^{2} \theta_{\mathrm{W}}=M_{\mathrm{W}}^{2} / M_{\mathrm{Z}}^{2}$ is a derived quantity. For the computation of the LO processes and the corresponding EW radiative corrections, we use the MSTW2008LO PDF set [37] in the LHAPDF setup [38]. In order to consistently include $\mathcal{O}(\alpha)$ corrections, in particular real radiation with the resulting collinear singularities, PDFs in principle should take these QED effects into account. Such a PDF analysis has been performed in Ref. [39], and the $\mathcal{O}(\alpha)$ effects are known to be small, as far as their effect on the quark distribution is concerned [40]. In addition, the currently available PDFs incorporating $\mathcal{O}(\alpha)$ corrections [39] include QCD effects at NLO, whereas our EW analysis is LO with respect to perturbative QCD only. For these reasons, the MSTW2008LO set is used as our default choice for the quark-induced processes. Our default choice for the factorization scale is the average of the vector-boson transverse masses

$\mu_{\mathrm{F}}=\overline{m_{\mathrm{T}}}=\frac{1}{2}\left(\sqrt{M_{V_{1}}^{2}+p_{\mathrm{T}, V_{1}}^{2}}+\sqrt{M_{V_{2}}^{2}+p_{\mathrm{T}, V_{2}}^{2}}\right)$

A similar scale choice was taken in Ref. [20] for the computation of the EW corrections to four-lepton production at the LHC. Yet we point out that the relative EW corrections, which are the main subject of this paper, only depend on the choice of $\mu_{\mathrm{F}}$ at the subpercent level even for large transverse momenta.

In our default setup, we require a minimum transverse momentum and a maximum rapidity for the final-state vector bosons,

$p_{\mathrm{T}, V_{i}}>15 \mathrm{GeV}, \quad\left|y_{V_{i}}\right|<2.5, \quad i=1,2$,

to define a $V$-boson pair production event. Thereby we exclude events where the bosons are emitted collinearly to the initial-state partons.

\footnotetext{
${ }^{1}$ We point out that a non-vanishing top-quark mass is consistently included in the computation of the one-loop contributions discussed in this paper.
}

In W-pair production, the invariant-mass distribution (Fig. 1 top) receives well-known logarithmically enhanced negative EW corrections ( $\left.\delta_{\mathrm{EW}}\right)$ growing with energy. Positive contributions arise from the partonic subprocess $\gamma \gamma \rightarrow \mathrm{WW}$ $\left(\delta_{\gamma \gamma}\right)$ and the photon-quark-induced processes $\left(\delta_{\gamma q}^{\mathrm{veto}}\right)$, the latter evaluated applying the dynamical jet veto also used in Ref. [7], where the transverse momentum of the jet has to be smaller than half of the leading-W $p_{\mathrm{T}}$. As already pointed out in Ref. [8], the effect of massive-boson radiation $\left(\delta_{3 V}\right)$ is moderate, however, strongly dependent on the event selection.

The above picture significantly changes if angular distributions of the $\mathrm{W}$-pair are studied at high invariant masses. This can be seen in Fig. 1 (bottom) where distributions of the rapidity gap of the two Ws are shown for $M_{\mathrm{WW}}>1,000 \mathrm{GeV}$. While the genuine EW corrections drastically reduce the differential cross section at high $p_{\mathrm{T}, \mathrm{W}}$, corresponding to small rapidity gap, the photon-induced contributions significantly increase the rate at small scattering angles, corresponding to large rapidity gap. As a result, a dramatic distortion of the angular distribution is visible which might easily be misinterpreted as signal of anomalous couplings.

We point out that the photon-induced corrections presented above (which are obtained using the MRST2004qed PDF set [39] for the photon density) suffer from a large systematic error stemming from our ignorance of the photon content of the proton. This becomes obvious looking at Fig. 25 of Ref. [41], where the NNPDF2.3QED [41] set has been used to estimate the error on the $\gamma \gamma$-induced $\mathrm{W}$-pair cross section. A relative error of $\pm 50 \%$ on the leading-order (LO) cross section at $M_{\mathrm{WW}}=1,000 \mathrm{GeV}$ can be deduced, solely induced by the photon PDF error. This picture indicates that a significant improvement in the determination of the photon PDFs is mandatory to reliably predict the W-pair production cross section at high energies.

Turning to $\mathrm{WZ}$ production, the situation is qualitatively similar to WW production, though here the $\gamma \gamma$ process is absent and the genuine EW corrections are smaller (Fig. 2). In Z-pair production, however, the $\gamma q$-induced contributions are negligible [23], and the real-radiation contributions are always below $10 \%$. In total, particularly large negative corrections, reaching $-40 \%$, dramatically affect Z-pair production at high invariant masses and transverse momenta (Fig. 3).

\section{$2.2 K$-factors for the electroweak corrections}

Let us start with the simplest reaction, inclusive Z-boson pair production. The full set of electroweak corrections, including photon radiation, Z-boson mass effects and virtual top quarks, has been discussed in Ref. [8], where the purely weak corrections were also evaluated for the 4-lepton final state in the pole approximation. The real and virtual QED 

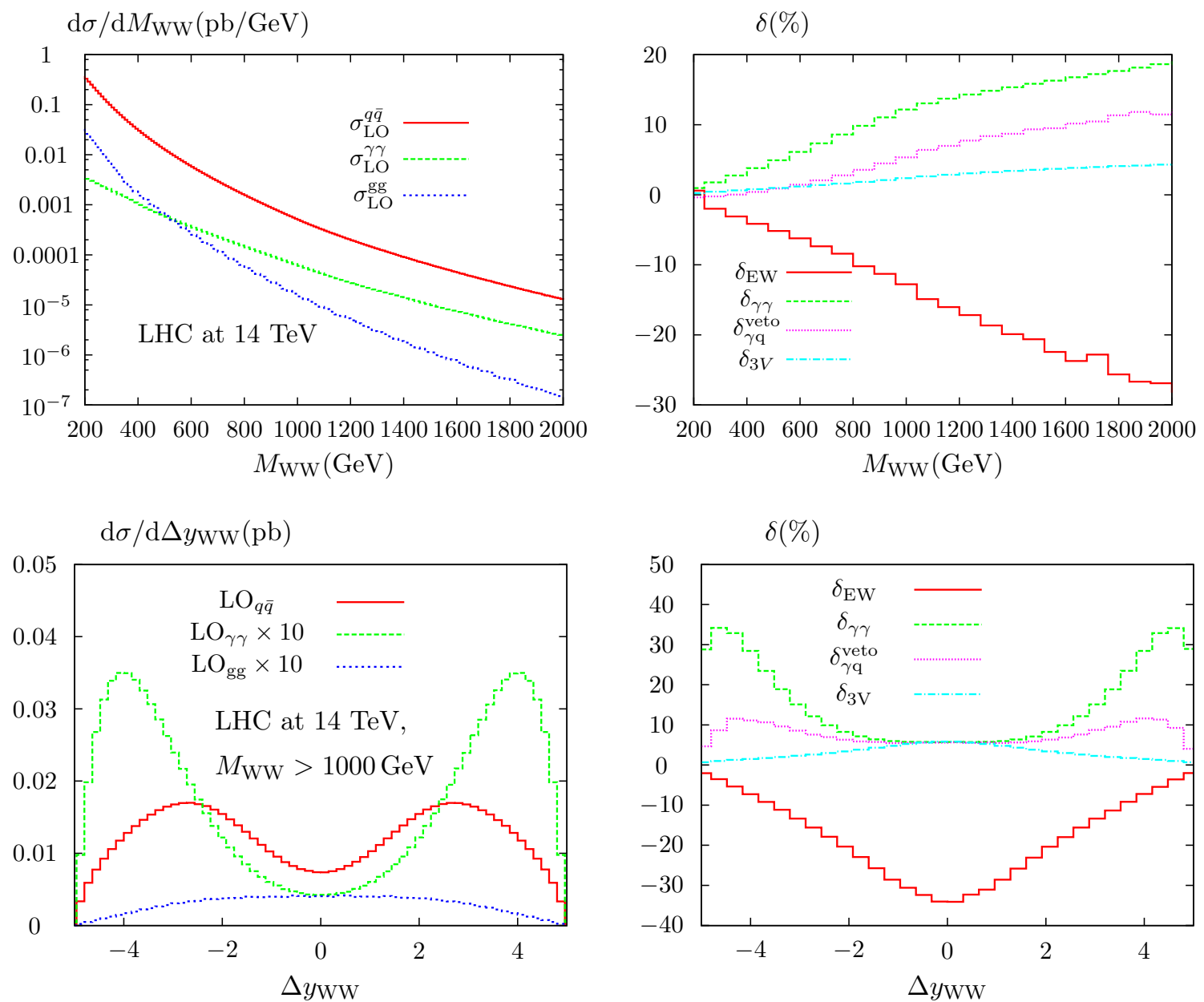

Fig. 1 Left differential LO cross sections for W-pair production at LHC14. Right various EW corrections relative to the quark-induced LO process. Top invariant-mass distribution; bottom WW rapidity-gap

distribution for $M_{\mathrm{WW}}>1 \mathrm{TeV}$. The results presented here are obtained in the default setup of Ref. [7]

corrections which can be considered as gauge invariant subset were included in the on-shell analysis. However, their contribution is relatively small, in general below $1 \%$. This is demonstrated in Fig. 4 for four characteristic distributions: the distributions in Z-boson rapidity and transverse momentum, in the invariant mass of the $\mathrm{Z}$ pair and, furthermore, in the difference between the rapidities of the two $\mathrm{Z}$ bosons. Note that in fact three correction functions are required, one for the uū induced reaction which we denote as

$K_{\mathrm{uu}}^{\mathrm{ZZ}}=1+\delta_{\mathrm{uu}}^{\mathrm{ZZ}}$,

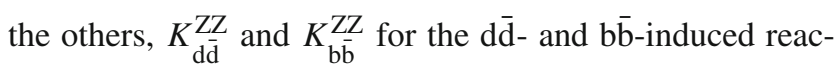
tions. All these factors depend on the partonic $\hat{s}$ and $\hat{t}$ only.

Similar considerations apply to di-photon production, and results analogous to $\mathrm{ZZ}$ production are also shown in Fig. 4. Again the relative contribution from purely photonic corrections is small and the two-body approximation reproduces the

full answer to better than $1 \%$. The correction factors $K_{\mathrm{uu}}^{\gamma \gamma}$, $K_{\mathrm{d} \overline{\mathrm{d}}}^{\gamma \gamma}$ and $K_{\mathrm{b} \overline{\mathrm{b}}}^{\gamma \gamma}$ are defined in an obvious way. The results presented in Fig. 4 were obtained using the default setup as defined in Ref. [8].

The situation is more involved in the case of W-pair and WZ production where weak and electromagnetic corrections are intimately intertwined. As is well known, virtual photonic corrections are required to arrive at an ultraviolet finite answer. The resulting infrared divergencies are then canceled by real radiation, the remaining collinear singularities are finally absorbed into a redefinition of the PDFs. Since one is now dealing partially with a three-body final state, the direct use of a $K$-factor which depends on $\hat{s}$ and $\hat{t}$ only is nontrivial.

In order to return to the kinematics of a two-to-two-body reaction, the corrections from real radiation are now replaced by just subtracting the endpoint singularities as defined originally in Ref. [42] and described in the appendix. As will be shown explicitly in Sect. 2.3, the physical predictions remain 

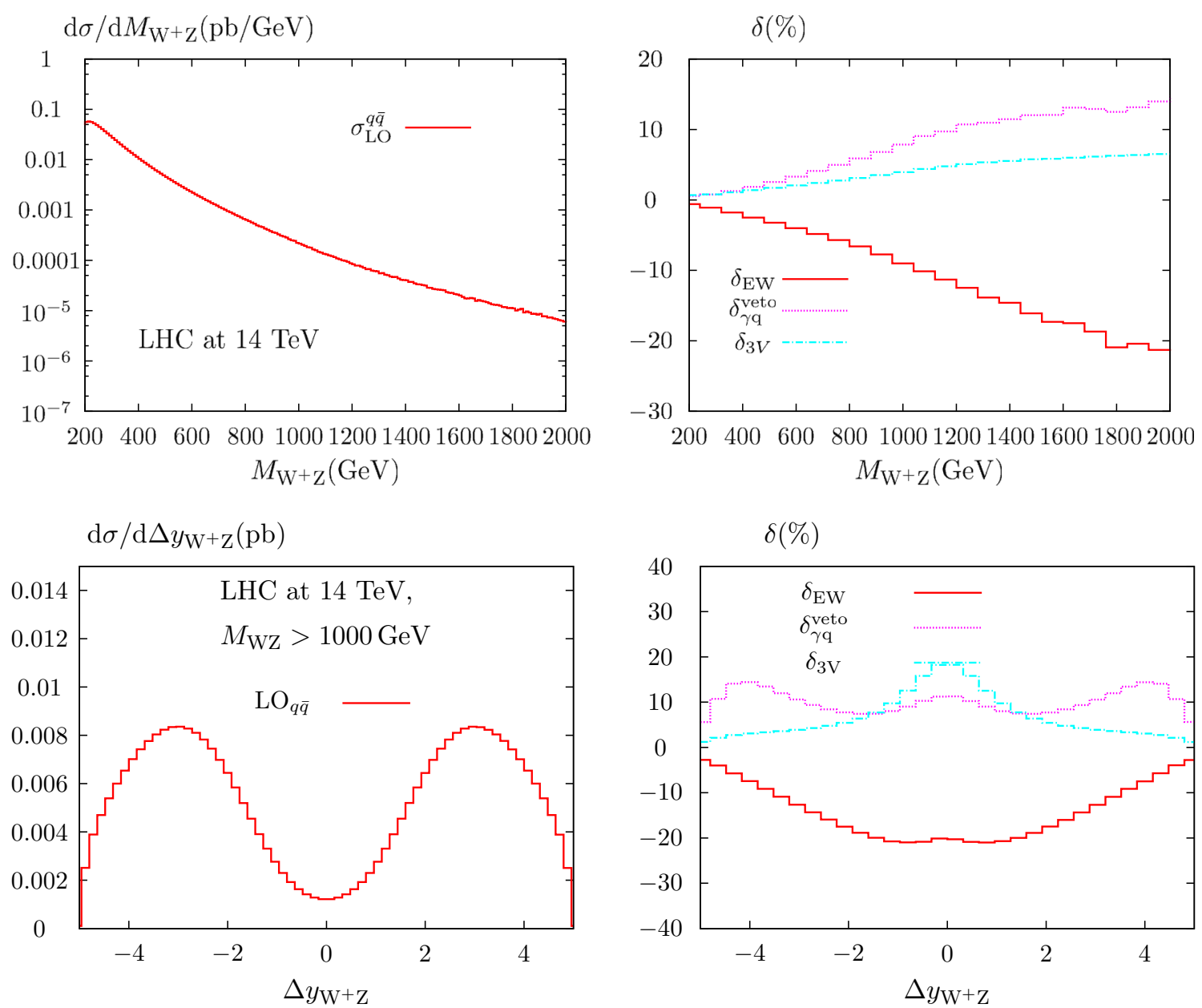

Fig. 2 Left differential LO cross sections for $\mathrm{W}^{+} \mathrm{Z}$ production at LHC14. Right various EW corrections relative to the quark-induced LO process. Top invariant-mass distribution; bottom WZ rapidity-gap

distribution for $M_{\mathrm{WZ}}>1 \mathrm{TeV}$. The results presented here are obtained in the default setup of Ref. [8]

practically unaffected by this simplification. (The agreement at the level of around one percent is demonstrated explicitly in Figs. 6 and 7 through the comparison between $\delta_{\mathrm{EW}}^{\text {full }}$ and $\delta_{\mathrm{EW}}^{\mathrm{V}+\mathrm{E}}$.) At the same time, the kinematics of the resulting final states is now fully described by the partonic $\hat{s}$ and $\hat{t}$. In this way we obtain again three correction factors $K_{\mathrm{uu}}^{\mathrm{WW}}, K_{\mathrm{d} \overline{\mathrm{d}}}^{\mathrm{WW}}$ and $K_{\mathrm{b} \overline{\mathrm{b}}}^{\mathrm{WW}}$ which can be employed in the framework of the Monte Carlo generator, just as before. A similar approach is valid for $\mathrm{W}^{+} \mathrm{Z}$ and $\mathrm{W}^{-} \mathrm{Z}$ production, which have, of course identical correction factors, denoted $K^{\mathrm{WZ}}$. The corresponding endpoint contributions are also listed in the appendix.

\subsection{Gauge-boson polarization and four-lepton production}

Our corrections are presented for unpolarized gauge bosons, which in the case of $\mathrm{W}$ and $\mathrm{Z}$ are observed through their decay products. The distributions of the decay products, however, are affected by the boson polarization. Hence an additional

complication could result from the fact that the polarization pattern of the gauge bosons is modified by the radiative corrections. In principle one would have to employ $K$-factors for the full set of helicity amplitudes. However, as demonstrated in Ref. [8] for ZZ production, in practice a fairly simple pattern emerges. Let us first consider the case of $\mathrm{Z}$ pairs: For small transverse momenta the electroweak corrections are small (about $-4 \%$ ) and of similar magnitude for all four combinations of transverse and longitudinal polarizations. For large transverse momenta one single configuration dominates completely and corrections for the subdominant combinations are irrelevant. This feature has been demonstrated in Table 7 of Ref. [8], where the cross sections and the corrections are displayed in the low-, intermediate- and large- $p_{\mathrm{T}}$ region, separated according to longitudinal and transverse polarizations.

From these considerations it becomes clear that in the case of Z-pair production a single partonic $K$-factor is sufficient for large as well as for small transverse momenta, 

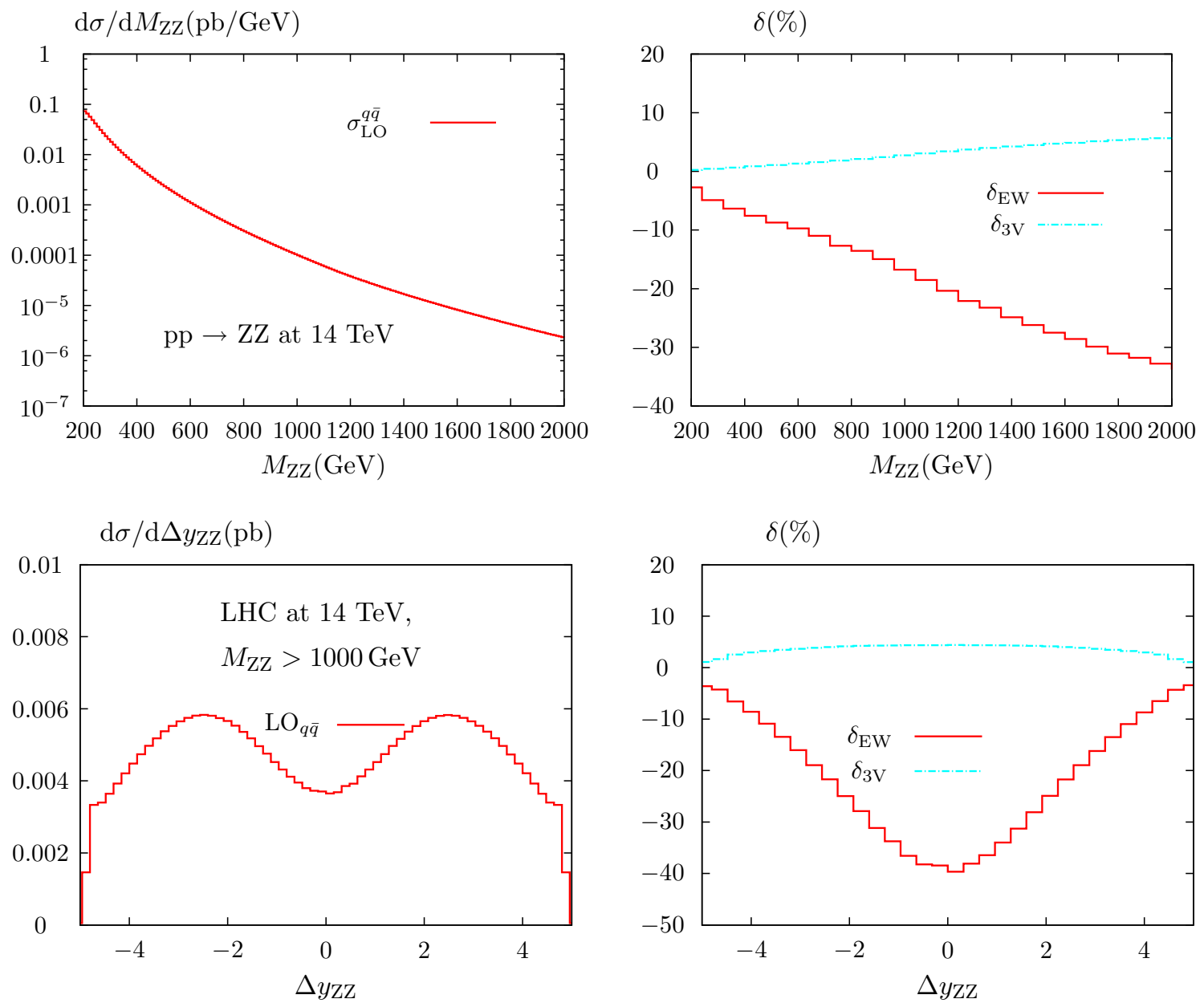

Fig. 3 Left differential LO cross sections for ZZ production at LHC14. Right various EW corrections relative to the quark-induced LO process. Top invariant-mass distribution; bottom $\mathrm{ZZ}$ rapidity-gap distribution for

$M_{\mathrm{ZZ}}>1 \mathrm{TeV}$. The results presented here are obtained in the default setup of Ref. [8]

and polarizations of the gauge bosons and, correspondingly, the correlations between the decay products as predicted in Born approximation are maintained even after inclusion of the electroweak corrections. The situation is slightly different for W-pair (and also WZ) production. Here, roughly $10 \%$ of the LO cross section are given by longitudinally polarized Ws even at high transverse momenta, and the corresponding relative EW corrections are substantially different compared to the transversely polarized case (see Fig. 7 of Ref. [22]). However, the numerical effects from the longitudinal polarization on the radiative corrections are small, and it is still sufficient - as will be shown later-to apply unpolarized $K$ factors to reproduce the full corresponding EW corrections with sufficient accuracy.

The corrections evaluated in Refs. [7,8] and encoded in our $K$-factors were obtained for on-shell Z or W bosons. Any realistic simulation of four-fermion production must, necessarily, include contributions from off-shell configurations. In the case of $\mathrm{Z}$ also diagrams with off-shell $\mathrm{Z}$ replaced by vir-

tual photons would be required for a description away from the $\mathrm{Z}$ peak. However, for the experimental analysis of gauge boson production the invariant mass of the decay products (lepton pairs or jets) must be restricted to an interval around the nominal mass, say $\left|M_{\bar{l}}-M_{\mathrm{Z}}\right|<25 \mathrm{GeV}$, to suppress the admixture of virtual photons and enhance close-to-massshell gauge bosons. In this case the neglect of virtual photons as implemented in HERWIG++ can be justified ${ }^{2}$.

In our approach off-shell effects and non-resonant contributions are consistently accounted for in the LO predictions, while at NLO the NWA is applied to compute the relative corrections. This approximation is well motivated, since offshell effects in the EW corrections only amount to $\sim 0.5 \%$, as demonstrated in Ref. [21] for W-pair production.

2 The HERWIG ++ implementation of $V$-pair production [43] relies on the double-pole approximation, where only doubly resonant contributions are taken into account including off-shell effects. 

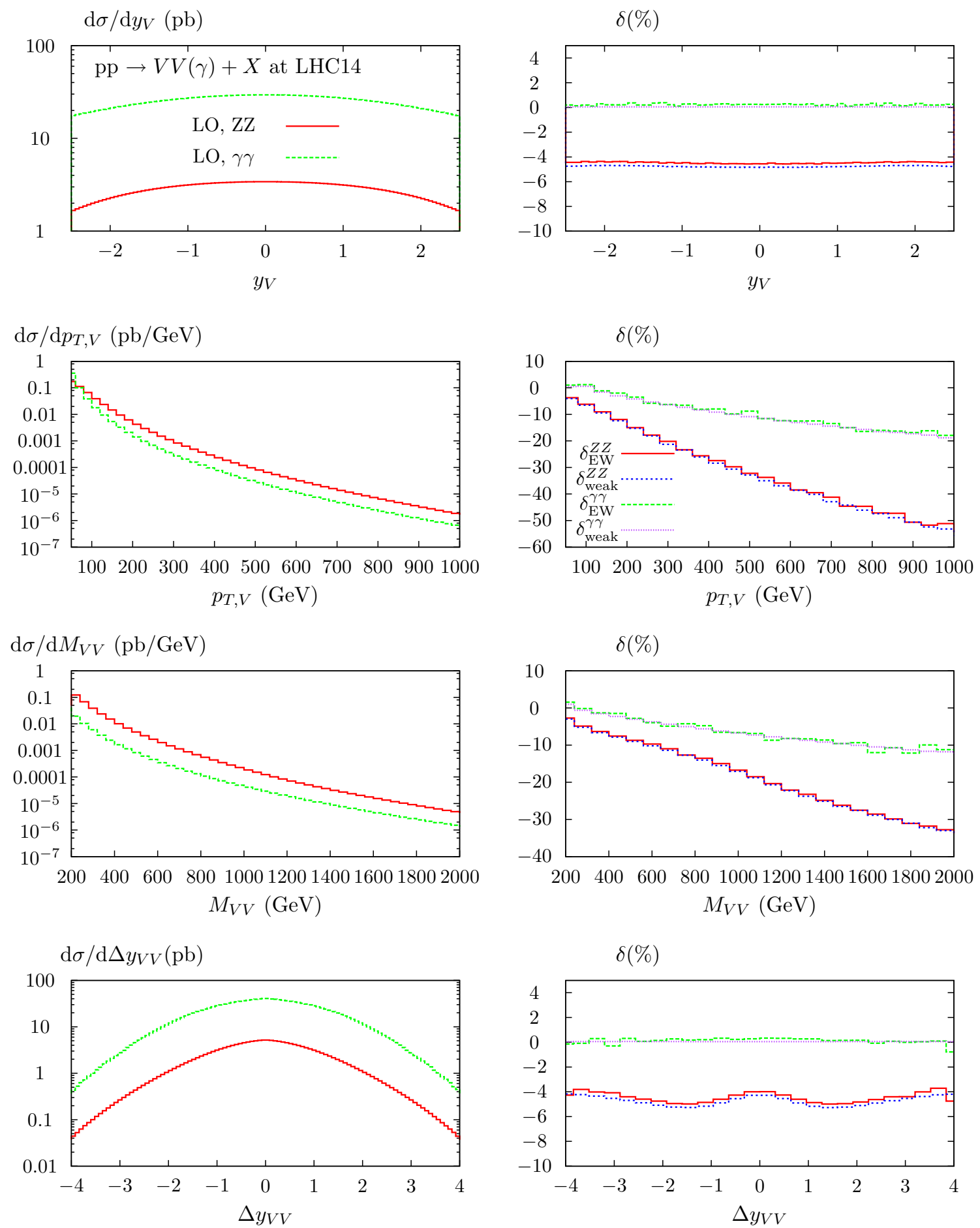

Fig. 4 Various differential distributions for on-shell Z-pair and $\gamma$-pair production at LHC14. Left LO predictions; right relative weak corrections $\left(\delta_{\text {weak }}^{V V}\right)$ and the full set of electroweak corrections $\left(\delta_{\mathrm{EW}}^{V V}\right)$, including

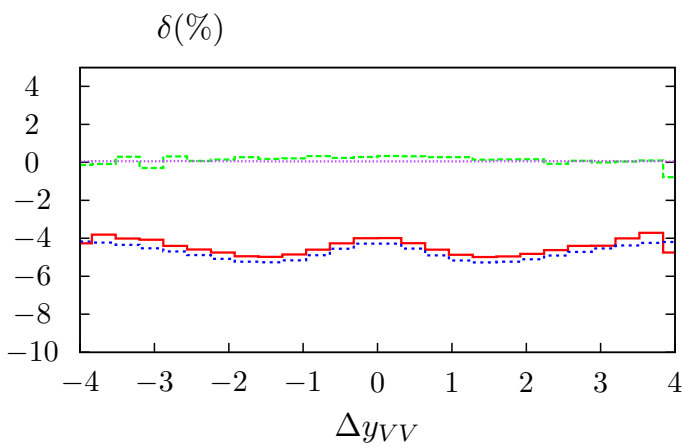

QED contributions. All results are obtained in the default setup defined in Ref. [8]

From these considerations it can be expected that the dominant weak corrections to four-lepton production at the LHC are well described by process dependent $K$-factors which can be taken from the unpolarized results for the corresponding

$2 \rightarrow 2$ production process. The validity of the approximations discussed above will now be studied in detail.

For this purpose, we give a concise presentation of the computation of EW corrections to massive gauge-boson pair 

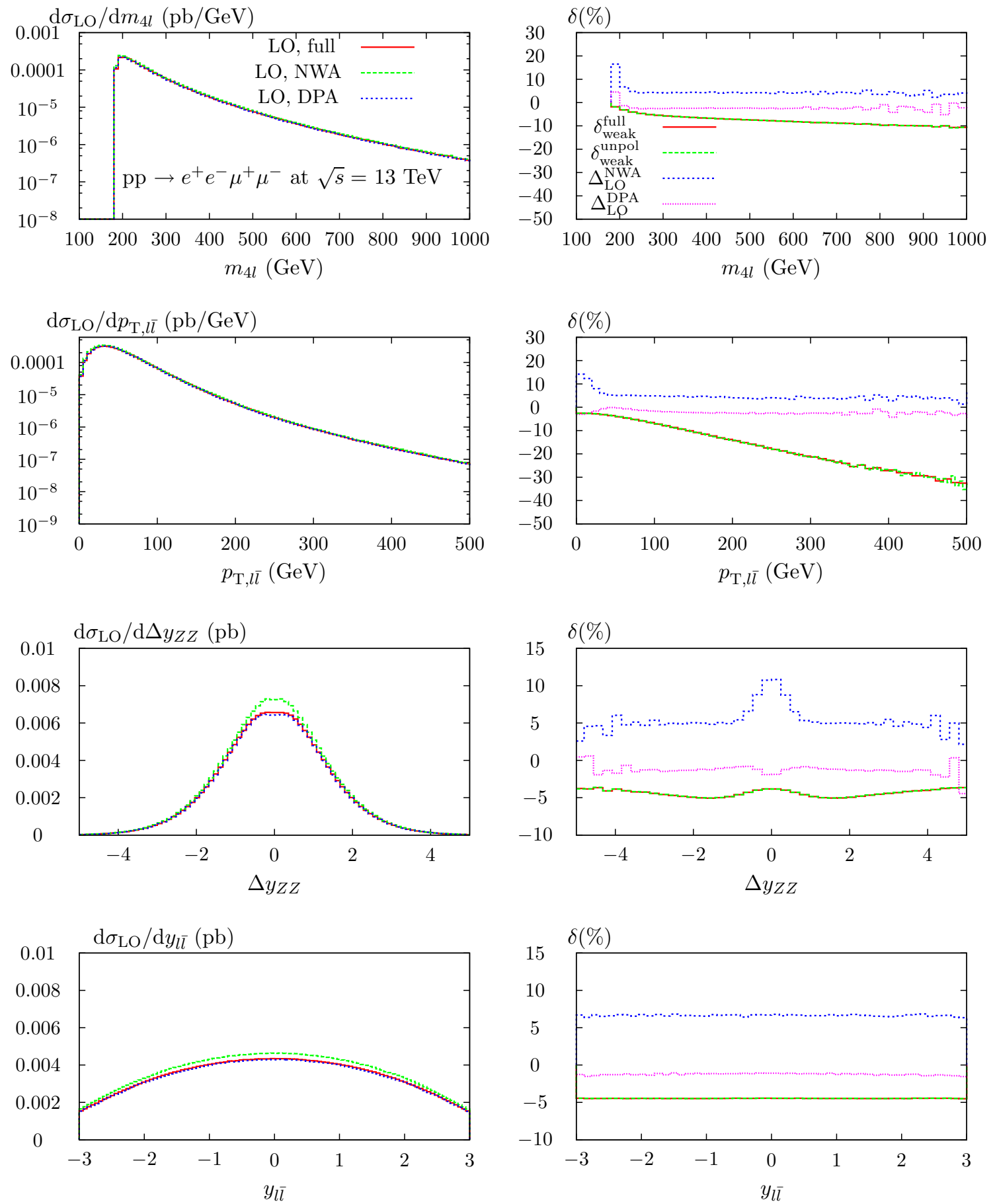

Fig. 5 Various differential distributions for $e^{+} e^{-} \mu^{+} \mu^{-}$production at LHC13. Left The full LO prediction as well as NWA and DPA are shown; right relative weak corrections $\delta_{\text {weak }}^{\text {full }}$ evaluated in the NWA, including

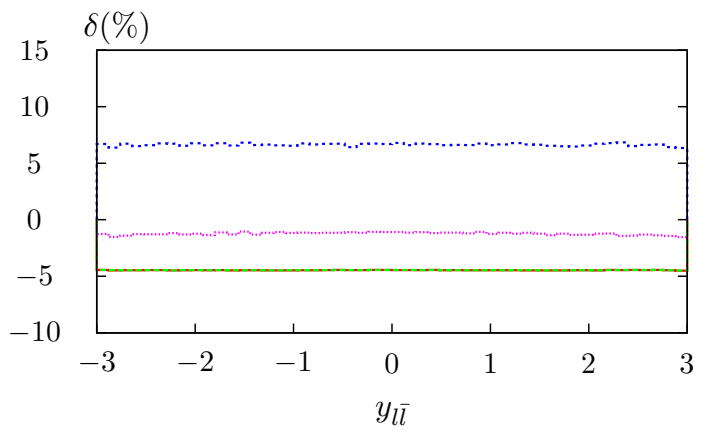

spin correlations; weak corrections evaluated with unpolarized $2 \rightarrow 2$ $K$-factors $\left(\delta_{\text {weak }}^{\text {unpol }}\right)$; relative deviations of NWA and DPA w.r.t. the full LO are also shown

production at the LHC, consistently taking into account leptonic decays and, related to this, spin correlations. In particular, we discuss the validity of the various approximations discussed in the previous subsection. To allow for a sensible comparison with the HERWIG++ results presented in Sect. 5, we generally stick to the default HERWIG++ setup for gauge-

boson pair production as defined in Ref. [43]. Specifically, in the leptonic event selection we apply the following basic cuts:

$p_{\mathrm{T}, l}>10 \mathrm{GeV}, \quad\left|y_{l}\right|<5$

for the charged-lepton transverse momenta and rapidities. If neutrinos are present in the final state, a minimal missing 

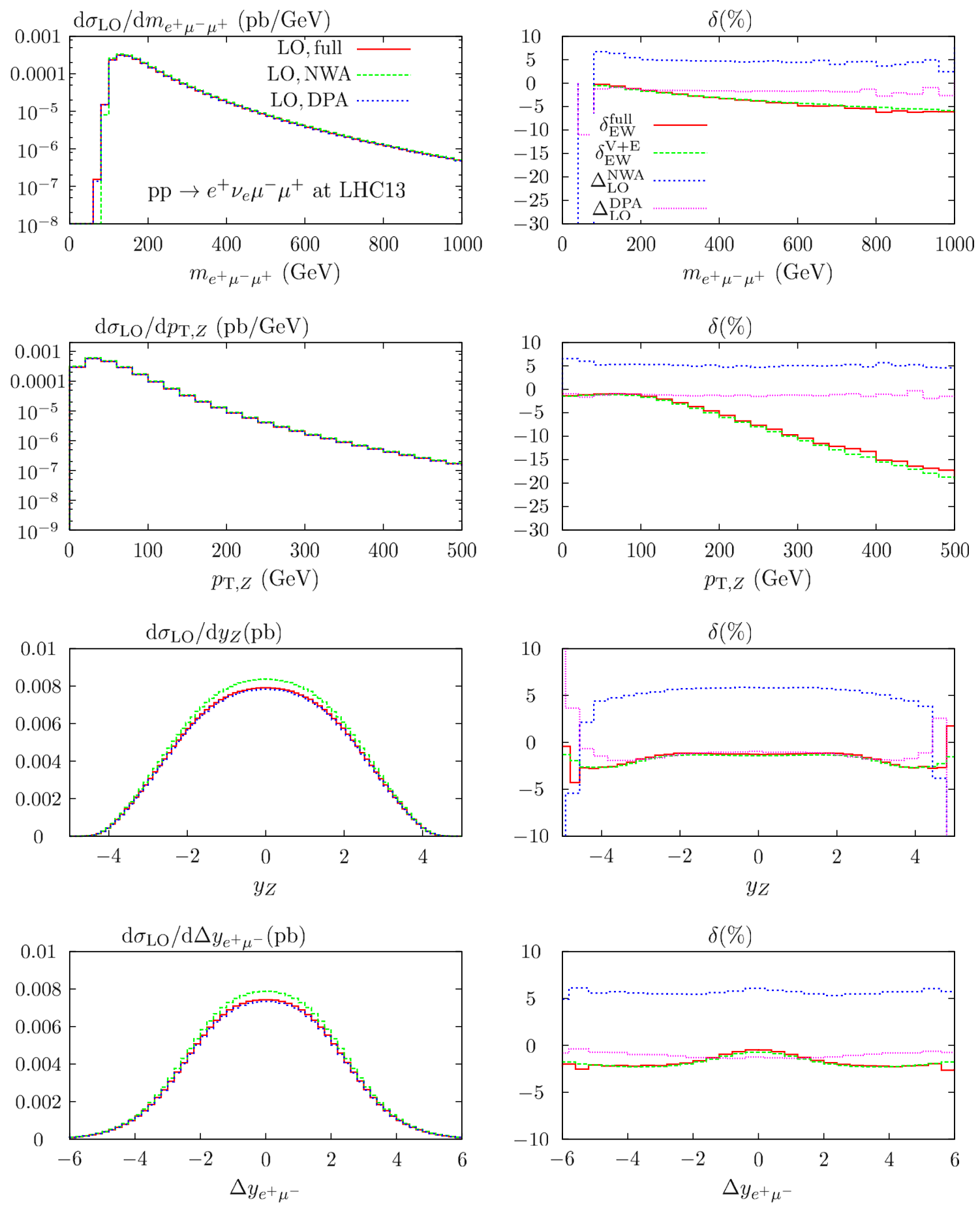

Fig. 6 Various differential distributions for $e^{+} v_{e} \mu^{-} \mu^{+}$production at LHC13. Left the full LO prediction as well as NWA and DPA are shown; right full relative EW corrections $\delta_{\mathrm{EW}}^{\text {full }}$ evaluated in the NWA including

spin correlations; EW corrections evaluated with unpolarized $2 \rightarrow 2 \mathrm{~K}$ factors in the $\mathrm{V}+\mathrm{E}$ approximation $\left(\delta_{\mathrm{EW}}^{\mathrm{V}+\mathrm{E}}\right)$; relative deviations of NWA and DPA w.r.t. the full LO are also shown

$\left|M_{l \bar{l}}-M_{V}\right|<25 \mathrm{GeV}$

transverse momentum of

$p_{\mathrm{T}, \mathrm{miss}}>25 \mathrm{GeV}$

is also required. The invariant mass of the lepton pair is restricted to to suppress non-resonant backgrounds. For the gauge-boson widths we use the values

$\Gamma_{\mathrm{W}}=2.141 \mathrm{GeV}, \quad \Gamma_{\mathrm{Z}}=2.4952 \mathrm{GeV}$, 

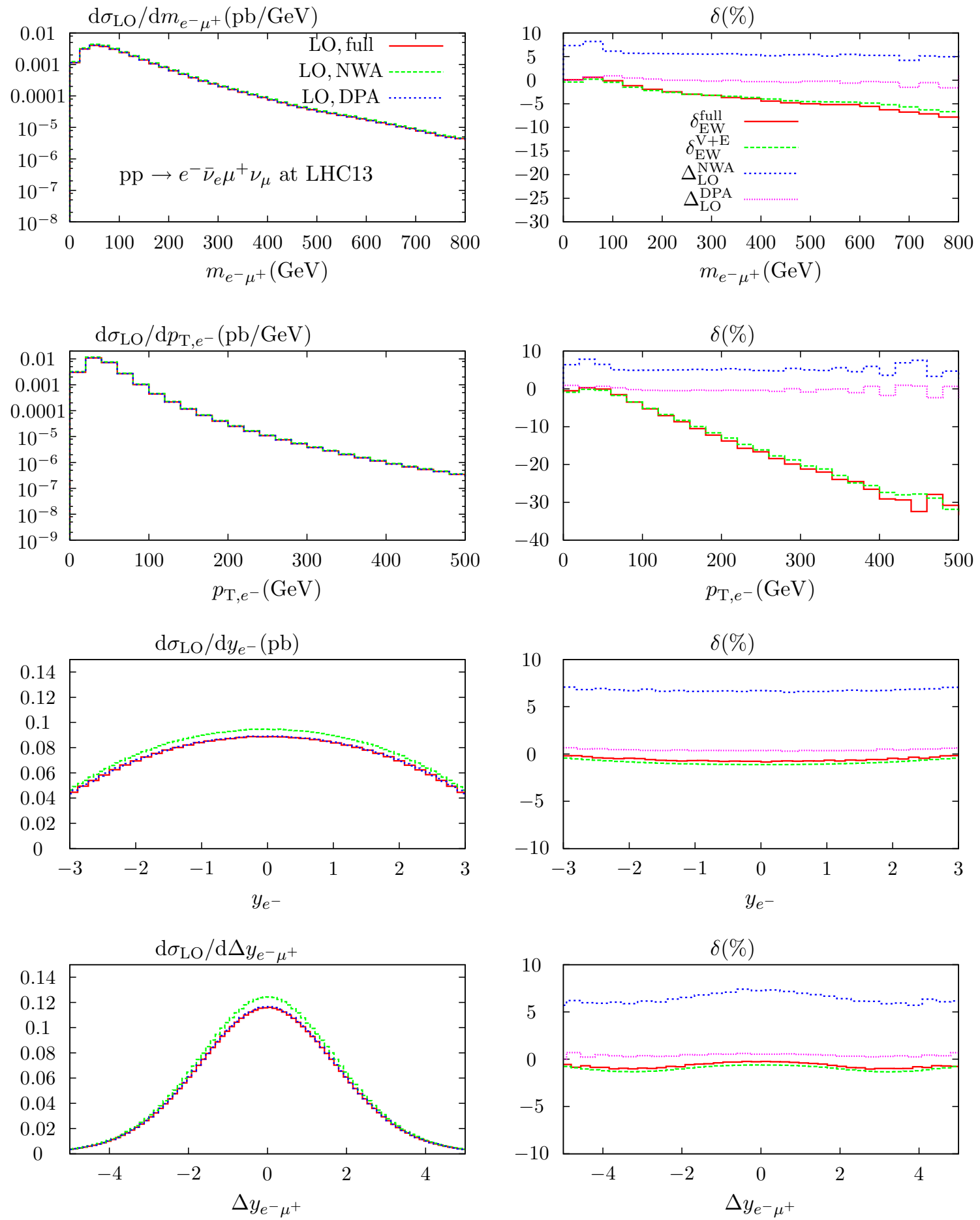

Fig. 7 Various differential distributions for $e^{-} \bar{v}_{e} \mu^{+} v_{\mu}$ production at LHC13. Left the full LO prediction as well as NWA and DPA are shown; right full relative EW corrections $\delta_{\mathrm{EW}}^{\text {full }}$ evaluated in the NWA including

and the weak coupling constant is defined in the $G_{\mu}$ scheme to systematically absorb universal corrections related to the running of $\alpha$ to the weak scale in the LO predictions. All remaining SM input parameters are directly carried over from Ref. [8].

spin correlations; EW corrections evaluated with unpolarized $2 \rightarrow 2 \mathrm{~K}$ factors in the $\mathrm{V}+\mathrm{E}$ approximation $\left(\delta_{\mathrm{EW}}^{\mathrm{V}+\mathrm{E}}\right)$; relative deviations of NWA and DPA w.r.t. the full $\mathrm{LO}$ are also shown

For the evaluation of hadronic cross sections we use the CT10NLO PDF set [44] in the LHAPDF framework [38], and the CKM dependence in the WZ production channels is included at leading order, while in the computation of EW corrections the CKM matrix is set to unity. 
At leading order we present full results, including nonresonant and off-shell effects, as well as results in two different approximations. As far as the full LO cross sections are concerned, we have checked that the difference between a naive fixed width implementation and results obtained in the Complex-Mass Scheme (CMS) $[45,46]$ is at the per-mill level and hardly visible. All results presented here for the full LO cross sections therefore correspond to the naive fixed-width implementation.

In addition to the full results, we provide the results for $V$-boson pair production in the double-pole approximation (DPA) originally discussed in Ref. [20]. Here, the amplitudes for V-pair production and decays are evaluated on-shell, but the Breit-Wigner shape of the resonance is included in the evaluation of the squared matrix elements to account for the dominant off-shell effects. We apply the on-shell projection procedure proposed in Ref. [47] to construct proper on-shell momenta of the intermediate bosons from the four-particle phase space. Note that in addition to the physical cuts displayed above, we impose a technical cut, $m_{4 l}>M_{V_{1}}+M_{V_{2}}$, on the 4-lepton invariant mass since the on-shell projection suggested in Ref. [47] only gives sensible results above threshold.

As a third alternative, we work in the narrow-width approximation (NWA), where the gauge bosons are strictly forced on-shell from the beginning via the replacement

$$
\frac{1}{\left(p^{2}-M_{V}^{2}\right)^{2}+M_{V}^{2} \Gamma_{V}^{2}} \rightarrow \frac{\pi}{M_{V} \Gamma_{V}} \delta\left(p^{2}-M_{V}^{2}\right)
$$

for the resonant squared propagators.

In Figs. 5, 6, and 7 we present results for $\mathrm{WW}, \mathrm{W}^{+} \mathrm{Z}$ and $\mathrm{ZZ}$ production at the LHC. Since in our approach the EW corrections to the partonic subprocesses are insensitive to the leptonic decay mode, in each case we concentrate on one specific decay channel, namely

$$
\begin{aligned}
& q \bar{q} \rightarrow\left(\mathrm{Z} / \gamma^{*}\right)\left(\mathrm{Z} / \gamma^{*}\right) \rightarrow \mathrm{e}^{+} \mathrm{e}^{-} \mu^{+} \mu^{-}, \\
& u_{i} \bar{d}_{j} \rightarrow \mathrm{W}^{+}\left(Z / \gamma^{*}\right) \rightarrow \mathrm{e}^{+} \nu_{\mathrm{e}} \mu^{-} \mu^{+}, \\
& q \bar{q} \rightarrow \mathrm{W}^{-} \mathrm{W}^{+} \rightarrow \mathrm{e}^{-} \bar{v}_{\mathrm{e}} \mu^{+} v_{\mu} .
\end{aligned}
$$

Note that if intermediate $\mathrm{Z}$ bosons are present in the process, the $\gamma^{*}$ contributions and all related interference contributions are taken into account in the full LO results, while those contributions are absent in DPA and NWA, respectively.

In the left panels of Figs. 5, 6 and 7 we present LO results for various typical differential distributions for processes (2.11) at LHC13, resepctively. Besides the full results, the respective approximate results in NWA and DPA are also shown, always taken in leading order.

The right-hand-side panels of the respective plots show the relative deviations of the NWA $\left(\Delta_{\mathrm{LO}}^{\mathrm{NWA}}\right)$ and DPA $\left(\Delta_{\mathrm{LO}}^{\mathrm{DPA}}\right)$ from the full results. For all pair-production channels one observes that the NWA overshoots the full LO predictions at the level of $5 \%$, while the DPA results give a good approximation valid at the 2-3\% level. From the upper right plot of Fig. 5, it becomes obvious that both approximate predictions become crude near the Z-pair production threshold where off-shell effects apparently become more important.

Let us now turn to the EW corrections. As stated before, we compute the full EW corrections to the respective polarized vector-boson pair-production processes, and include the spin correlations in the decays to leptons, which are treated at leading order. The actual computation is carried out in the well-established FeynArts/FormCalc/LoopTools [48-53] setup already used in Refs. [7,8], and Madgraph [54] was useful for internal checks. We do not take into account the EW corrections to the leptonic decay processes for various reasons. On the one hand, the bulk of the EW corrections to $\mathrm{Z}$ and $\mathrm{W}$ decay are given by final-state photon radiation (FSR), which leads to significant distortions of the phase-space distributions of the leptonic decay products. These FSR corrections, however, are included in HERWIG ++ [55] in the YFS framework [56] and will therefore not be considered here. On the other hand, electroweak corrections to the inclusive boson decay widths are implicitly included in the experimentally determined values for the branching ratios used in the HERWIG++ framework. Additionally, we strictly stick to the NWA for the computation of the EW corrections. In this simplified approach, the corrections completely factorize into corrections either to the production or the decay process. No non-factorizable corrections, connecting production and decay, have to be considered. Those contributions have to be taken into account using the DPA as demonstrated in Ref. [20]. However, it is well known that the non-factorizable corrections largely cancel in sufficiently inclusive observables [57].

In addition to the full $\mathrm{EW}$ corrections $\delta_{\mathrm{EW}}^{\text {full }}$ (which contain proper spin correlations and, in the case of WW and WZ production, also the full set of QED corrections to the respective production process) approximate results $\delta_{\mathrm{EW}}^{\mathrm{V}+\mathrm{E}}$, employing the virtual+endpoint $(\mathrm{V}+\mathrm{E})$ approximation, are presented in the same plots. In this case unpolarized on-shell $K$-factors have been used to obtain the relative corrections, as detailed in Sect. 2.2. One observes that the approximate ansatz gives an almost perfect approximation for the full result, in general better than $1 \%$. The best agreement is observed for $\mathrm{ZZ}$ production, while for WW production a slight discrepancy is visible. This can be understood recalling that photon radiation and related QED corrections are largest for WW production, as has been demonstrated in Ref. [8], while they remain small in the case of $\mathrm{Z}$ pairs. In general, the agreement between the full result and the $\mathrm{V}+\mathrm{E}$ approximation is even better than expected. As a conclusion one finds that it is justified to use unpolarized $K$-factors in the $\mathrm{V}+\mathrm{E}$ approximation to describe vector-boson pair production at the LHC at sufficient accuracy. 


\section{Electroweak and QCD corrections: combined}

As stated in the introduction, the complete treatment of combined QCD and electroweak corrections, involving e.g. twoloop terms of order $\alpha_{s} \alpha_{\text {weak }}$ is presently out of reach. Consequently neither an additive treatment of the corrections, $\left(1+\delta_{\mathrm{QCD}}+\delta_{\text {weak }}\right)$, nor a multiplicative one, $\left(1+\delta_{\mathrm{QCD}}\right)(1+$ $\left.\delta_{\text {weak }}\right)$, will lead to the correct result. However, the bulk of the QCD corrections for the processes under consideration is related to soft or collinear gluon radiation from the incoming quark-antiquark system. Soft radiation leaves the initial state practically unchanged, the quarks remain close to their mass shell and the weak corrections can be taken from the $\hat{s}$ and $\hat{t}$ dependent $K$-factor.

A similar line of reasoning is applicable to hard collinear radiation which leads to final states with vanishing or small transverse momentum of the diboson system. Let us assume that a collinear gluon is radiated from the incoming (anti-)quark, which subsequently initiates the boson pair production. Also in this case the (anti-)quark stays close to its mass shell. The scattering angle of the $q_{1} \bar{q}_{2} \rightarrow V_{1} V_{2}$ reaction in the $V_{1} V_{2}$ rest frame can again be directly identified with the scattering angle of the partonic reaction. A similar line of reasoning applies to reactions with quarks or antiquarks originating from gluon splitting.

The situation becomes more involved in the case of hard non-collinear radiation which leads to a diboson system of large transverse momentum. In this case only an approximate prescription can be formulated, since the original $2 \rightarrow 2$ kinematics is distorted. However, this approximate prescription must coincide with the previous one in the limit of vanishing transverse momentum. To be specific, we advocate the following strategy to compute the effective partonic Mandelstam variables $\hat{s}^{\prime}$ and $\hat{t}^{\prime}$ from the distorted kinematics for the evaluation of $K\left(\hat{s}^{\prime}, \hat{t}^{\prime}\right)$ : The squared CM energy is calculated from the four-lepton final state via

$\hat{s}^{\prime}=m_{4 l}^{2}$.

The momenta are boosted into the four-lepton CM frame (denoted by $\Sigma^{*}$ ). In this frame the unit directions of initialstate hadrons shall be denoted by

$\vec{e}_{i}^{*}=\frac{\vec{p}_{i}^{*}}{\left|\vec{p}_{i}^{*}\right|}, \quad i=1,2$.

The direction of the effective scattering axis in $\Sigma^{*}$ is now defined by

$\hat{\vec{e}}^{*}=\frac{\vec{e}_{1}^{*}-\vec{e}_{2}^{*}}{\left|\vec{e}_{1}^{*}-\vec{e}_{2}^{*}\right|}$,

and the effective scattering angle is, correspondingly, given by

$\cos \theta^{*}=\vec{v}_{1}^{*} \cdot \hat{\vec{e}}^{*}$, where the $\vec{v}_{i}^{*}$ denotes the momentum direction of vector boson $V_{i}$. The Mandelstam variable $\hat{t}^{\prime}$ is then computed from $\theta^{*}$ assuming on-shell kinematics.

Diboson events with large transverse momenta necessarily require the presence of at least one hard quark or gluon jet, and electroweak corrections would have to be evaluated separately for this class of processes. As long as they can be treated as a small admixture to the diboson sample, suppressed by an additional factor $\alpha_{s}$, the distortion of the weak corrections should not lead to a significant error for the inclusive sample. If one is interested specifically in the analysis of the diboson process, a cut on the transverse momentum of the dibosons system will eliminate the pollution with events of a very different nature. Let us discuss this important issue in some more detail. As pointed out by several groups [7,15], at large transverse momenta $V$-pair production is dominated by new topologies which are absent at lowest order in QCD. These topologies correspond to $V+$ jet production with the radiation of an additional $V$ from the quark jet rather than being a correction to $V$-pair production and spoil the perturbative series for the prediction of leptonic observables at high transverse momenta. They lead to huge QCD $K$-factors together with large residual scale uncertainties. To improve the corresponding theory predictions the authors of Ref. [15] have provided approximate NNLO QCD predictions for these particular observables in WZ production, applying the LoopSim method [58]. They observe pronounced shifts of the predictions going from NLO to NNLO and at the same time a significant reduction of the theory uncertainties. In this work, however, we follow another strategy to stabilize the problematic high- $p_{\mathrm{T}}$ observables, employing suitable restrictions on the transverse momentum of the 4-lepton system, as will be detailed in Sect. 5 .

In the present section a fairly general prescription has been suggested how to implement weak corrections into a generic Monte Carlo program which includes QCD corrections and hadronization already. Indeed this prescription allows for an $a$ posteriori application of EW corrections to any QCD Monte Carlo, at least as long as the partonic origin (uū vs. $d \bar{d})$ of the final state remains under control. In the next section a slightly different approach will be described which is specifically tailored to the program HERWIG++. In this case a partonic $\hat{s}$ and $\hat{t}$ is introduced in the program from the very beginning and, in the limit of diboson events with small transverse momenta, coincides with the prescription described above. In the following section this correspondence will be investigated in more detail.

\section{Implementation into HERWIG++}

Our starting point of the implementation of the EW corrections in HERWIG++ is the electroweak $K$-factor. 
Following the strategy of multiplicative QCD and EW corrections we reweight the events that have been generated in HERWIG ++ as the hard processes. For vector-boson pair production HERWIG++ delivers unweighted events. Hence we can compute $K(\hat{s}, \hat{t})$ and use this directly as a reweighting factor for each event such that $K(\hat{s}, \hat{t})<1$ leads to a suppression of events with given $(\hat{s}, \hat{t})$ while $K(\hat{s}, \hat{t})>1$ leads to an enhancement. The actual implementation of a reweighting factor for a given hard event is straightforward in HERWIG++ and ThePEG once the variables $(\hat{s}, \hat{t})$ are known.

Let us discuss the kinematics of our events in more detail. For EW corrections to the Born process the calculation of a $K$-factor is straightforward as we can directly access the kinematic setup of the hard process once this is generated. In this case $(\hat{s}, \hat{t})$ can be computed uniquely. As soon as we want to apply the EW corrections to an event that is generated from an NLO QCD matrix element this is no longer the case. In this case we face the complication that an event with real radiation is not described by $2 \rightarrow 2$ kinematics anymore.

Fortunately, the situation in the HERWIG + + case is largely simplified by the fact that the NLO QCD corrections are applied in the POWHEG scheme [43,59]. (See $[60,61]$ for more details). The POWHEG scheme provides a consistent matching of NLO corrections and parton shower emissions without double counting. In this scheme, the differential cross section for the hard process can be written as

$$
\begin{aligned}
\mathrm{d} \sigma= & \bar{B}\left(\Phi_{B}\right) \mathrm{d} \Phi_{B}\left[\Delta_{R}\left(k_{\perp, \min }\right)+\frac{R\left(\Phi_{B}, \Phi_{R}\right)}{B\left(\Phi_{B}\right)}\right. \\
& \left.\times \Delta_{R}\left(k_{\perp}\left(\Phi_{B}, \Phi_{R}\right)\right) \mathrm{d} \Phi_{R}\right] .
\end{aligned}
$$

Here, $\Phi_{B}$ and $\Phi_{R}$ denote the Born and radiative phase space variables, respectively, and $k_{\perp, \min }$ is the minimum transverse momentum that is generated by the parton shower. $\Phi_{R}$ only parametrizes the additional variables to specify a hard emission relative to the Born configuration. $B\left(\Phi_{B}\right)$ and $R\left(\Phi_{R}\right)$ are the Born and real-emission matrix elements squared for the hard process under consideration. $B\left(\Phi_{B}\right)$ is the Born differential cross section, while

$$
\bar{B}\left(\Phi_{B}\right)=B\left(\Phi_{B}\right)+V\left(\Phi_{B}\right)+\int R_{S}\left(\Phi_{B}, \Phi_{R}\right) \mathrm{d} \Phi_{R} .
$$

Here, $V\left(\Phi_{B}\right)$ is the (infrared finite) sum of virtual corrections and the divergent part of the real corrections, while $R_{S}\left(\Phi_{B}, \Phi_{R}\right)$ is the (also finite) real correction matrix element squared with the divergent terms subtracted. Finally, the POWHEG Sudakov form factor is given by

$$
\begin{aligned}
\Delta_{R}\left(p_{\perp}\right)=\exp [ & -\int \mathrm{d} \Phi_{R} \frac{R\left(\Phi_{B}, \Phi_{R}\right)}{B\left(\Phi_{B}\right)} \\
& \left.\times \Theta\left(k_{\perp}\left(\Phi_{B}, \Phi_{R}\right)-p_{\perp}\right)\right],
\end{aligned}
$$

which, opposed to the Sudakov form factor in a common parton shower, contains the full real-emission matrix element squared. The two terms in (4.1) are constructed to resemble the result of a single parton shower emission applied to a configuration $\Phi_{B}$ with weight $\bar{B}\left(\Phi_{B}\right)$. The first term gives the no-emission probability, the second the contribution from a single emission. At the same time, upon expansion in $\alpha_{s}$, (4.1) reproduces the differential cross section at NLO QCD.

In the HERWIG++ implementation we find exactly this prescription. In a first step we generate an event with kinematical configuration $\Phi_{B}$. Technically, this is already the hard process. Only in a second step, already as part of the parton shower algorithm, the potential hard emission with relative kinematics $\Phi_{R}$ is generated according to the Sudakov form factor (4.3). Once this is done, the default parton shower is used as at this point, all terms will become formally higher than next-to-leading order and the default parton shower is computationally much simpler than the Sudakov form factor (4.3).

As the hard emission from POWHEG is technically already part of the parton shower, we have direct access to the Born-type variables $\Phi_{B}$ in the hard subprocess and hence we may easily compute the kinematic variables $(\hat{s}, \hat{t})$ for every event. In effect, this assumes that the EW corrections are applied only on the level of Born-type kinematics and are not strongly influenced by the hard emission. In fact, by applying a suitable veto, we later also focus our analysis to regions where the transverse momenta generated by hard gluon emissions are not too large. This veto will suppress events where gauge-boson pairs are accompanied by additional hard quark or gluon jets, leaving the $q \bar{q}$ events largely unaffected. This also enforces the kinematics to be reasonably close to a Born configuration in order to justify our approach.

Events with strong QCD activity, e.g. jets with large transverse momentum give rise to large $\mathrm{QCD}$ corrections. In order to suppress these enhanced corrections [58], we apply an additional cut on the final state in our analysis. Focusing on the leptonic final state, we have to make sure that the gauge-boson pairs or its decay products, the four leptons, are not produced with too much transverse momentum from the recoil against a system of strongly interacting particles. We achieve this by applying a condition on the leptons' transverse momenta $\vec{\ell}_{i, \mathrm{~T}}$ in the laboratory frame,

$$
\left|\sum_{i} \vec{\ell}_{i, \mathrm{~T}}+\vec{p}_{\mathrm{T}, \mathrm{miss}}\right|<\rho\left(\sum_{i}\left|\vec{\ell}_{i, \mathrm{~T}}\right|+\left|\vec{p}_{\mathrm{T}, \mathrm{miss}}\right|\right) .
$$

Here, we consider all visible leptons $i$ from EW boson decays, i.e. $i$ runs to 2, 3 or 4 in the case of $\mathrm{WW}, \mathrm{WZ}$ or $\mathrm{ZZ}$ events. We additionally assume that the missing transverse momentum in the event solely stems from neutrinos from W decays. The left-hand-side of (4.4) is small whenever the 

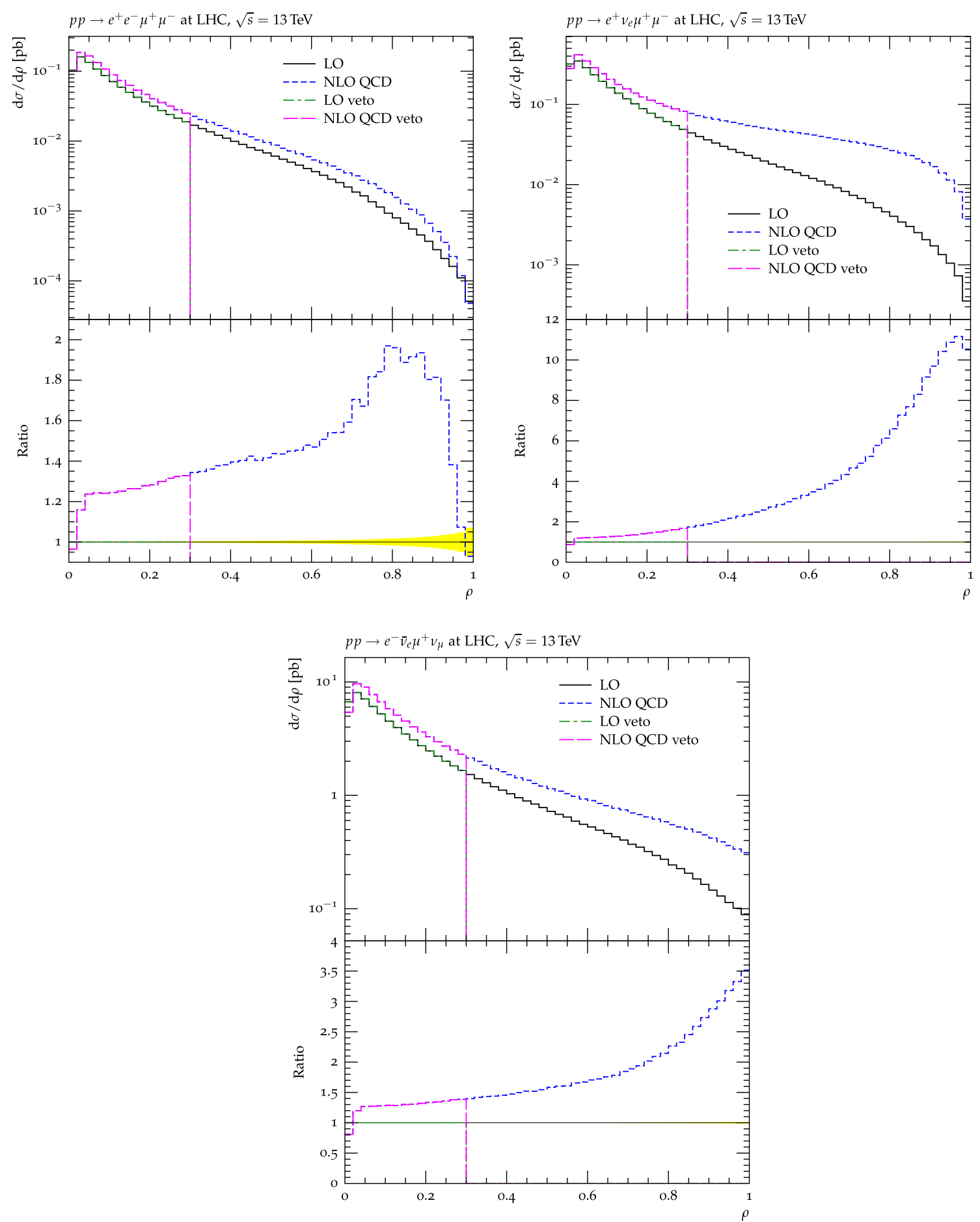

Fig. 8 Histograms in the veto variable $\rho=\left|\sum_{i} \vec{\ell}_{i, \mathrm{~T}}+\vec{p}_{\mathrm{T}, \mathrm{miss}}\right| /\left(\sum_{i}\left|\vec{\ell}_{i, \mathrm{~T}}\right|+\left|\vec{p}_{\mathrm{T}, \text { miss }}\right|\right)$ for the three different final states under consideration. For $\rho<0.3 \mathrm{LO}$ and LO veto coincide, as well as NLO QCD and NLO QCD veto

gauge-boson pair has a small transverse momentum, and the leptons from the EW system recoil against each other. After some experimenting, we find that $\rho=0.3$ gives a good selection of events while leaving enough events for our analysis at the same time.
In Fig. 8 we show histograms of the ratio $\rho$ of vector and scalar sums of lepton transverse momenta that we finally apply the cut on. We show runs with and without NLO QCD corrections. We find that the chosen value $\rho=0.3$ is a sensible choice for all three combinations of boson pairs. 

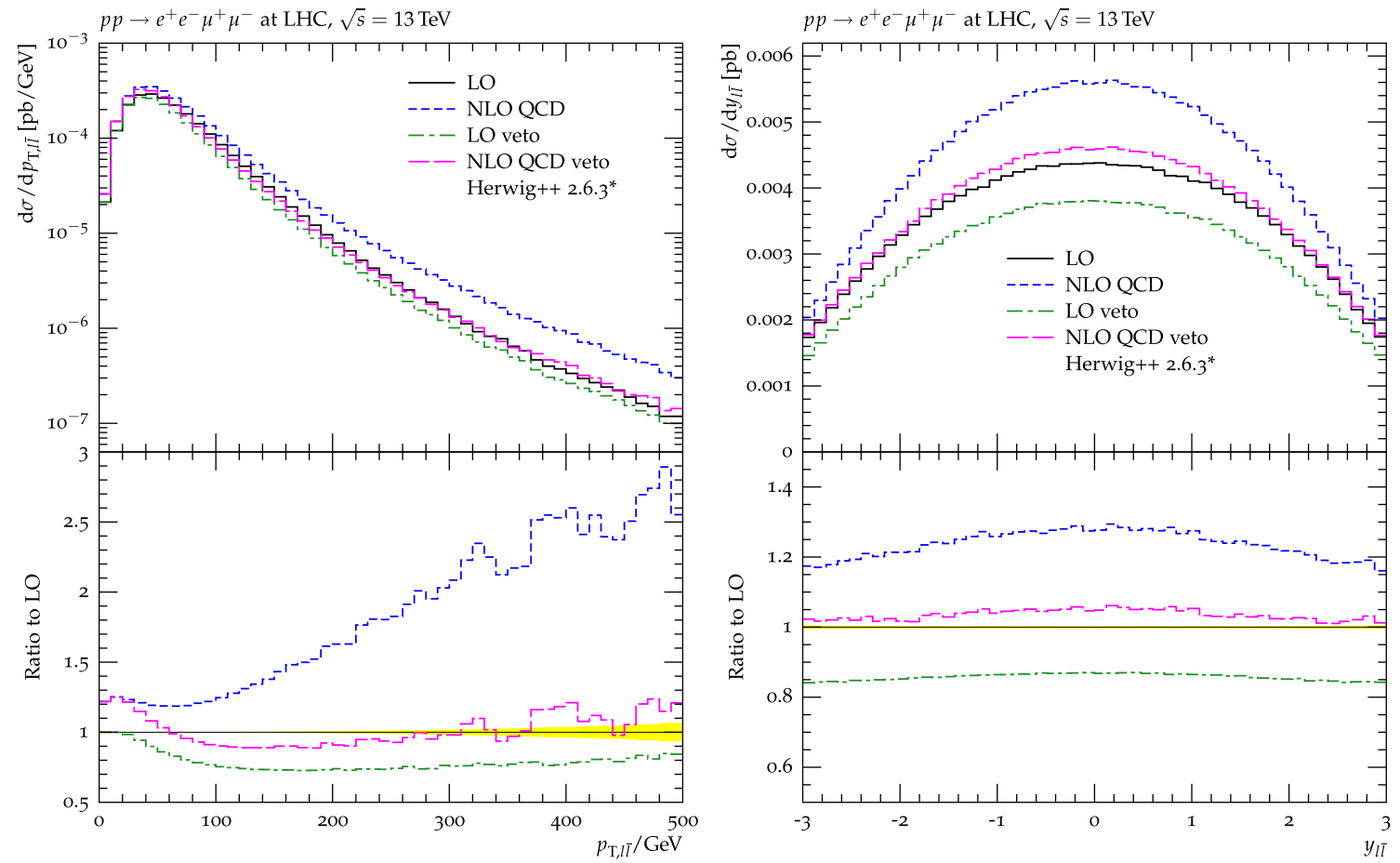

Fig. $9 \mathrm{ZZ}$ production at LHC13, effect of veto on selected leptonic observables

A good number of events is still available for the analysis while at the same time we find that the events with large distortions from hard radiation are vetoed with our selection. These are peaked at large values of the ratio in all three cases. In a full experimental analysis the value of $\rho$ might be subject to optimization for the individual cases of vector-boson pairs.

In addition we show actual leptonic observables with and without application of the lepton veto in Figs. 9, 10, 11. Let us compare LO and NLO predictions for the $p_{\mathrm{T}, \bar{l}}$ distribution in ZZ production (Fig. 9, left) which corresponds essentially to the transverse-momentum distribution of the $\mathrm{Z}$ boson. Without cut the NLO distribution exceeds the LO distribution by a factor of 2 at $p_{\mathrm{T}, l \bar{l}}=300 \mathrm{GeV}$ and more at larger transverse momenta. Introducing the cut (4.4) with $\rho=0.3$ removes most of this excess such that the difference between LO and NLO distributions is reduced to $\mathcal{O}(20 \%)$ and remains relatively constant as a function of $p_{\mathrm{T}, l \bar{l}}$. The ratio between the NLO and LO rapidity distribution, in contrast, is fairly constant with a ratio of $\mathrm{NLO} / \mathrm{LO} \sim 1.2$, and is reduced to $\sim 1$ by the cut for $\rho=0.3$. A similar behavior is observed for WZ (Fig. 10) in the $e^{+} v_{e} \mu^{+} \mu^{-}$mode with a giant correction factor of 4 for transverse momenta of the $\mathrm{Z}$ boson of $300 \mathrm{GeV}$ and a correction between 1.4 and 1.6 for the rapidity distribution. As before, one obtains a significant reduction of the QCD correction down to $\mathcal{O}(20 \%)$ by introducing the cut (4.4), and a similar behavior is observed for $\mathrm{W}^{+} \mathrm{W}^{-}$production in the $e^{-} \bar{v}_{e} \mu^{+} v_{\mu}$ channel (Fig. 11). In total, after applying the cut, LO and NLO results are close over the whole range in transverse momentum and rapidity.

Let us now estimate the effect of the veto (4.4) on the residual uncertainties due to missing higher-order QCD effects. For this purpose, we vary the renormalization and factorization scale around its central value, using $\mu_{F}=\mu_{R}=\xi M_{V V}$, with the rather conservative choice $10^{-1}<\xi<10$. In Fig. 12, exemplaric results for scale-variation effects in Zpair production at NLO with and without veto are presented. One finds that the resulting error bands are not significantly affected by the veto. Similar results are also found for WW and $\mathrm{WZ}$ production.

In order to assess the sensitivity to the choice of partonic kinematic variables we have studied the impact of $K(\hat{s}, \hat{t})$ with two additional choices for the kinematical variables for $\mathrm{ZZ}$ production, as in this case we have full access to the kinematics of the final state.

Let us call the variables from the direct access to the kinematics within HERWIG++ before the parton shower $\left(\hat{s}_{\text {hard }}, \hat{t}_{\text {hard }}\right)$. As an alternative, we reconstruct the kinematics from the vector-boson final state as outlined in the previous section. Here, no knowledge of the initial-state partons is needed for the computation of the kinematic invariants. $\hat{s}_{\text {rec }}$ is given by the invariant mass of the vector-boson pair and $\hat{t}_{\text {rec }}$ is reconstructed from the scattering angle in the centerof-mass frame as proposed in (3.1)-(3.4). For illustration, we also consider a choice of variables that is very likely to be wrong. We take the initial-state partons after the termina- 

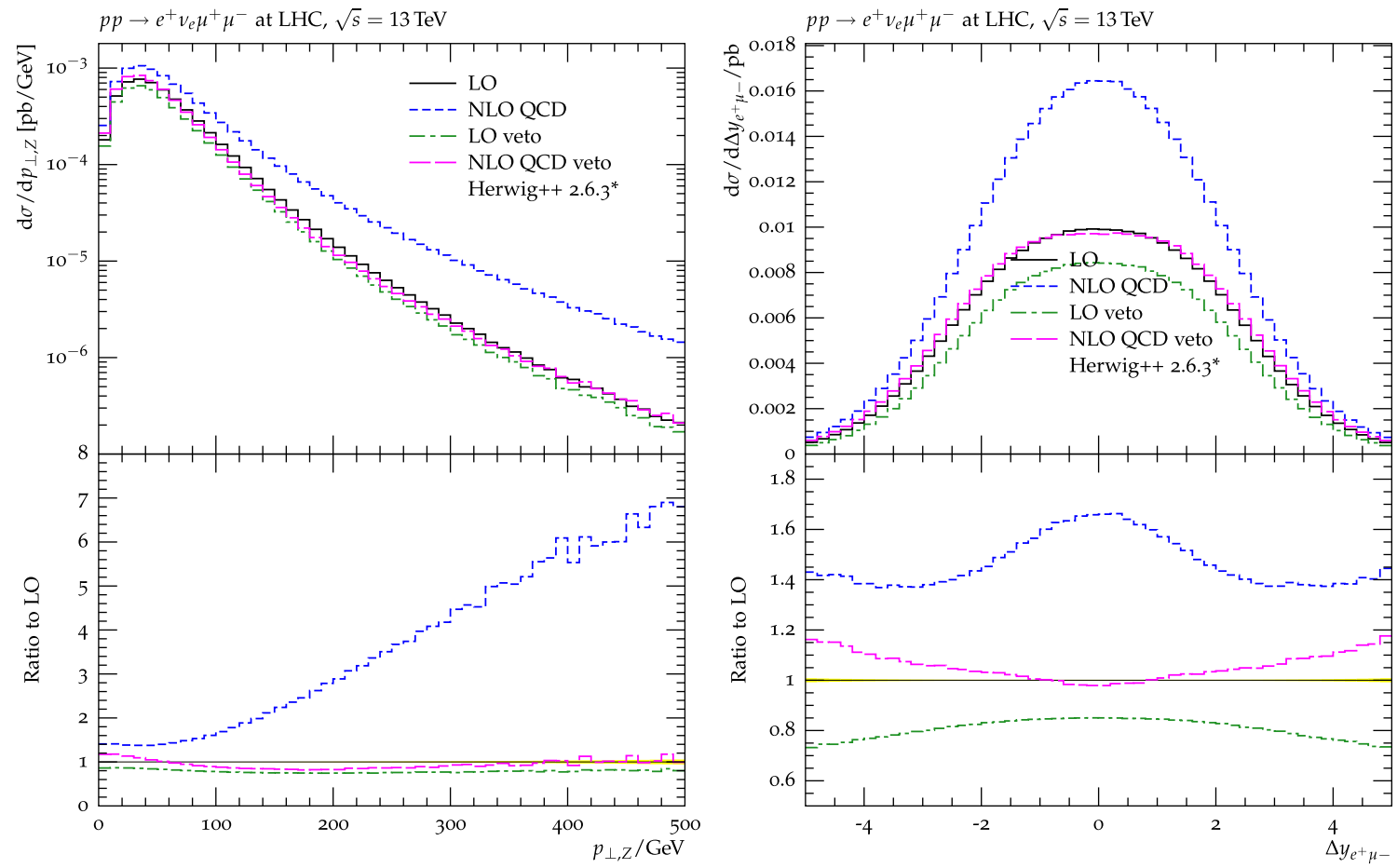

Fig. 10 WZ production at LHC13, effect of veto on selected leptonic observables
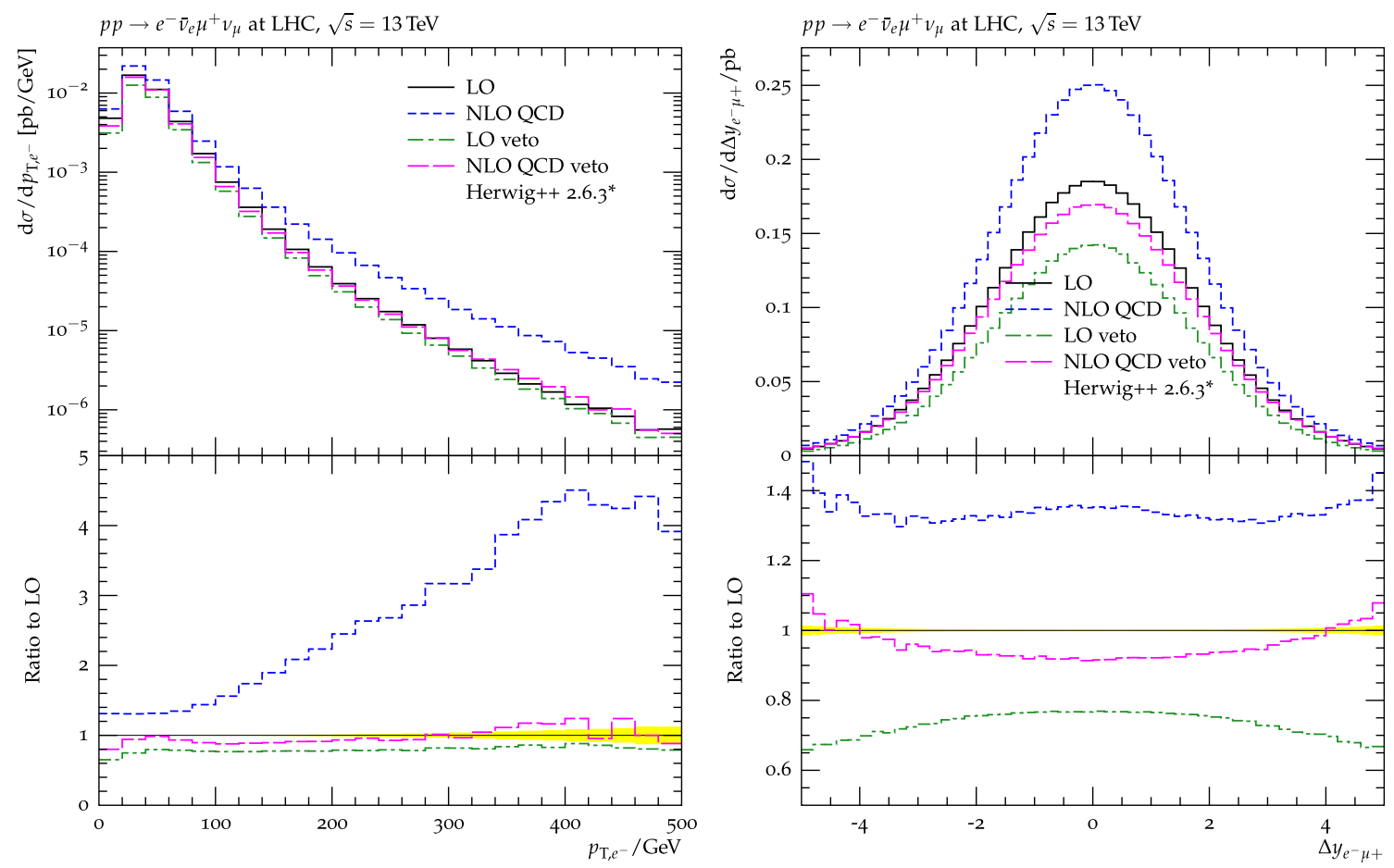

Fig. 11 WW production at LHC13, effect of veto on selected leptonic observables

tion of the parton shower. This parton pair will have a much larger invariant mass $\hat{s}_{\mathrm{PS}}$ due to the parton showering. We then find the four momenta of the outgoing vector bosons and compute $\hat{t}_{\mathrm{PS}}$ with respect to this initial state. This last choice will demonstrate the sensitivity to the parton shower emissions. For each event produced by HERWIG++ we con- 

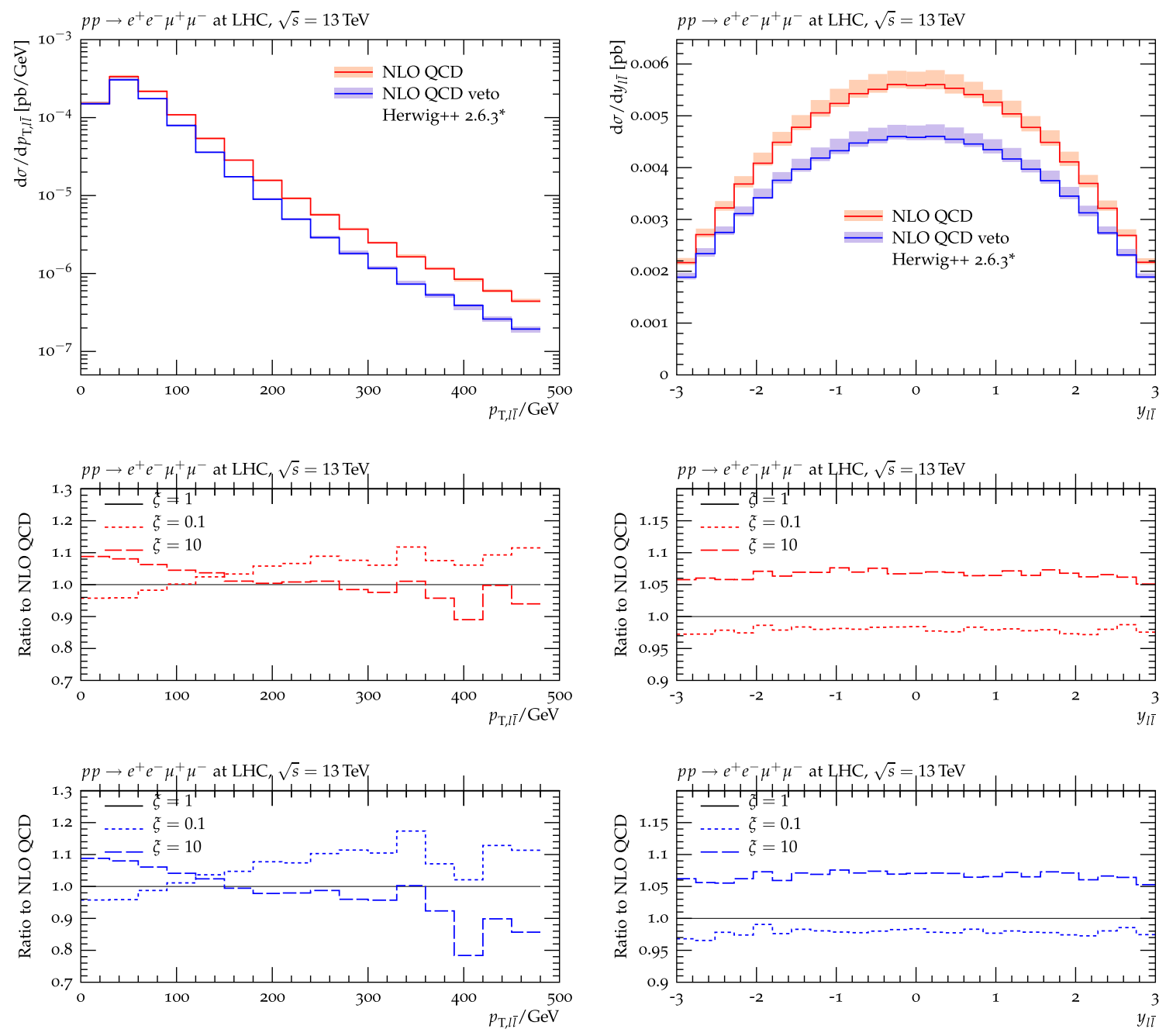

Fig. 12 Z-pair production at LHC13. Residual QCD uncertainties are estimated via variation of the (identified) renormalization and factorization scale. The bands shown in the plots are obtained using $\mu_{F}=\mu_{R}=\xi M_{\mathrm{WW}}$, with $10^{-1}<\xi<10$. Results with (blue)

and without (red) the veto (4.4) are displayed, where the ratios are normalized to the central value of the respective NLO QCD prediction

struct three sets of kinematic variables $\left(\hat{s}_{i}, \hat{t}_{i}\right)$ with $i \in$ \{hard, rec, PS\}. Then we compute the three different $K$ factors $K_{i}$ from these three sets of variables. Taking $K_{\text {hard }}$ as a reference we compute the ratio $K_{i} / K_{\text {hard }}$ for each event. If the reconstruction would be insensitive to the choice of kinematics this ratio ought to be unity all the time.

In Fig. 13 we show the distribution of this ratio for the two different ratios $K_{\mathrm{rec}} / K_{\text {hard }}$ and $K_{\mathrm{PS}} / K_{\text {hard }}$. As expected, the results from the reconstruction after the application of the parton shower are quite different from unity and show a very broad peak. This is reasonable and demonstrates that a wrong choice of kinematic variables leads to important differences in the computation of the $K$-factor.

In strong contrast, the EW $K$-factors based on the kinematics reconstruction from the final state compared to the $K$-factor based on the variables of the hard process inside the event generator are very close. Their ratio is very strongly

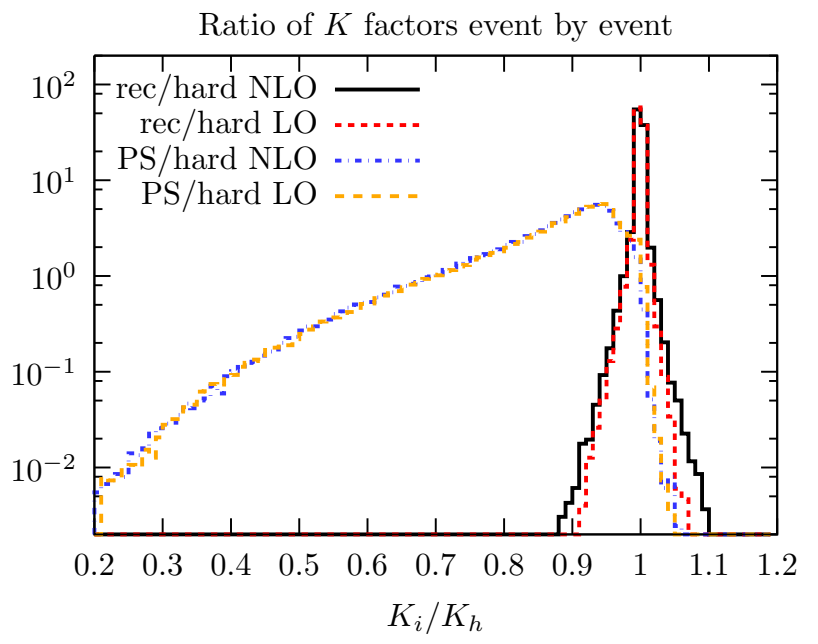

Fig. 13 The ratio of $K$-factors for different reconstructions $i$ of the kinematic variables $\left(\hat{s}_{i}, \hat{t}_{i}\right)$ in Z-pair production at LHC13. See text for further explanation 

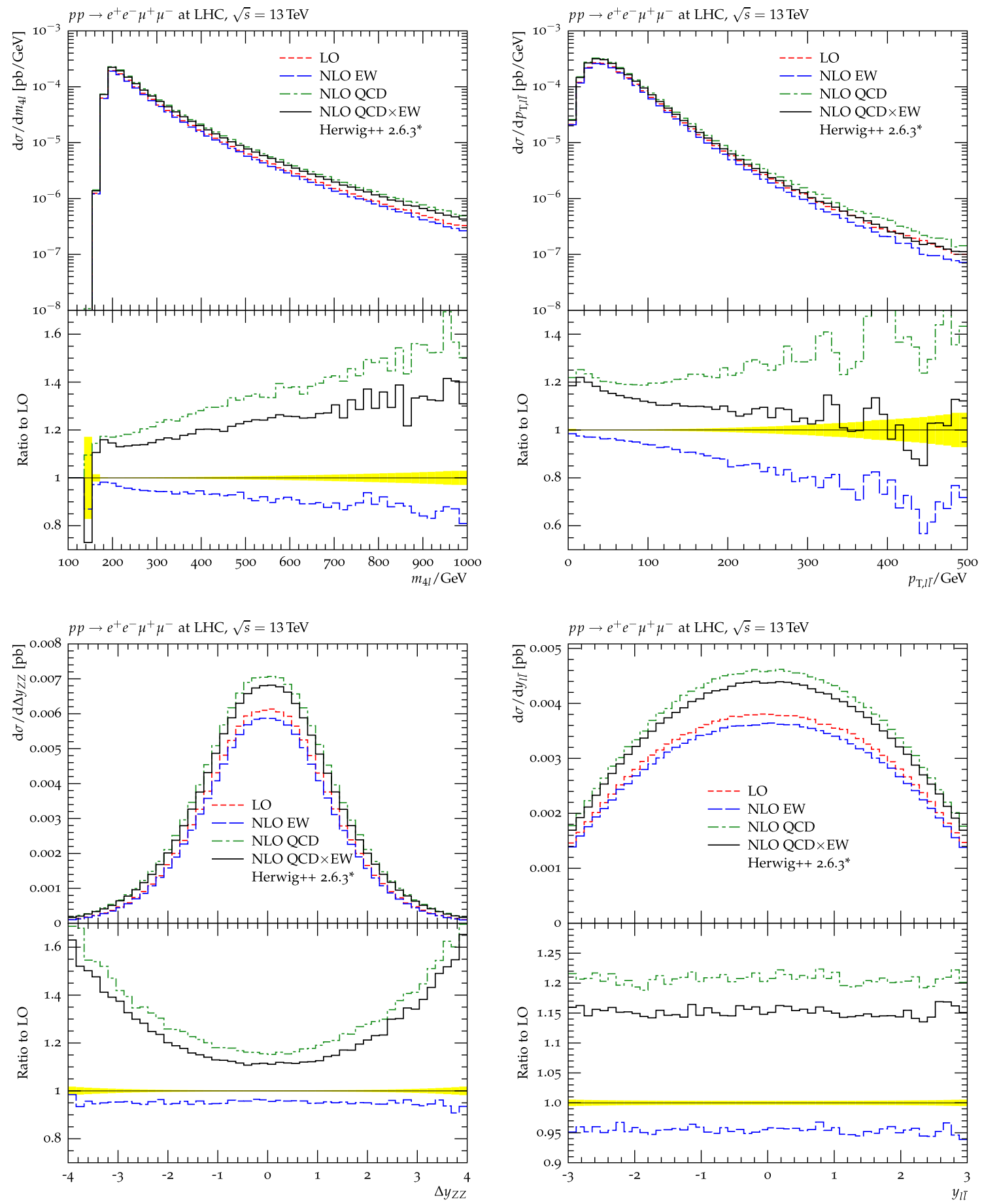

Fig. 14 Results from $\mathrm{ZZ}$ events at $\sqrt{s}=13 \mathrm{TeV}$. We show observables that can be reconstructed from the leptonic final state after we applied the veto (4.4)

peaked around one, demonstrating that the two prescriptions lead to nearly identical results.

In the same Fig. 13 we compare the results from runs with and without NLO QCD corrections. In both cases we get very similar results for the EW $K$-factor. The additional hard gluon from the real-emission graph distorts the kine- matics that was reconstructed in the leading-order case only slightly. As expected the QCD corrections lead to slightly bigger difference between the two reconstruction schemes.

We conclude that our computation of the EW correction is very robust against small variations of the kinematics as long as the variables are sensibly chosen and a sensible veto 

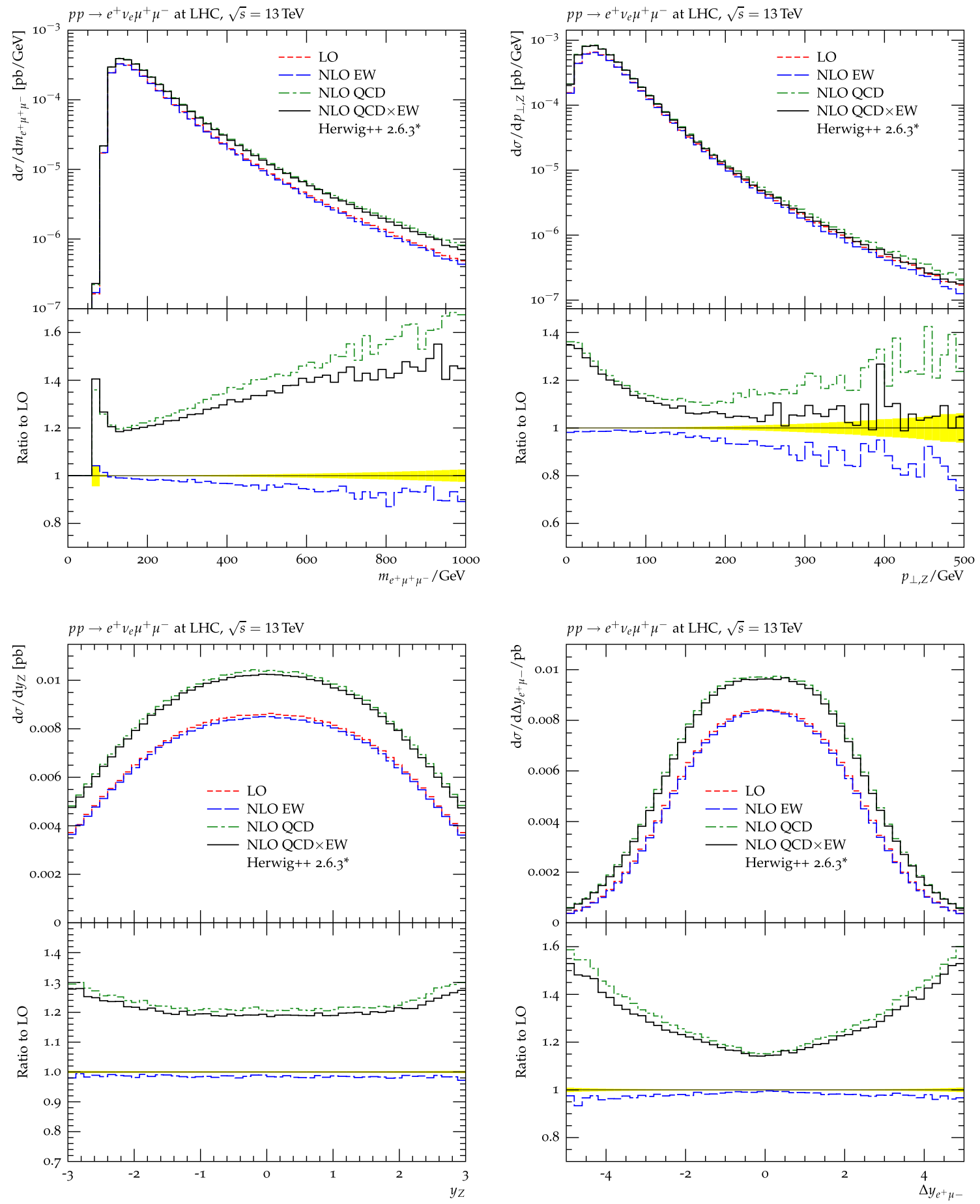

Fig. 15 Results from WZ events at $\sqrt{s}=13 \mathrm{TeV}$. We show observables that can be reconstructed from the visible leptonic final state after we applied the veto (4.4)

is applied. The reconstruction of kinematics from the final state alone, as proposed in the previous section, is a viable choice. In contrast, naively ignoring the parton shower in the reconstruction of kinematics will lead to significantly different results.

\section{HERWIG++ results}

Before we discuss the phenomenology of EW corrections in the HERWIG++ framework in detail, we would like to stress again that the multiplicative approach combining EW and 

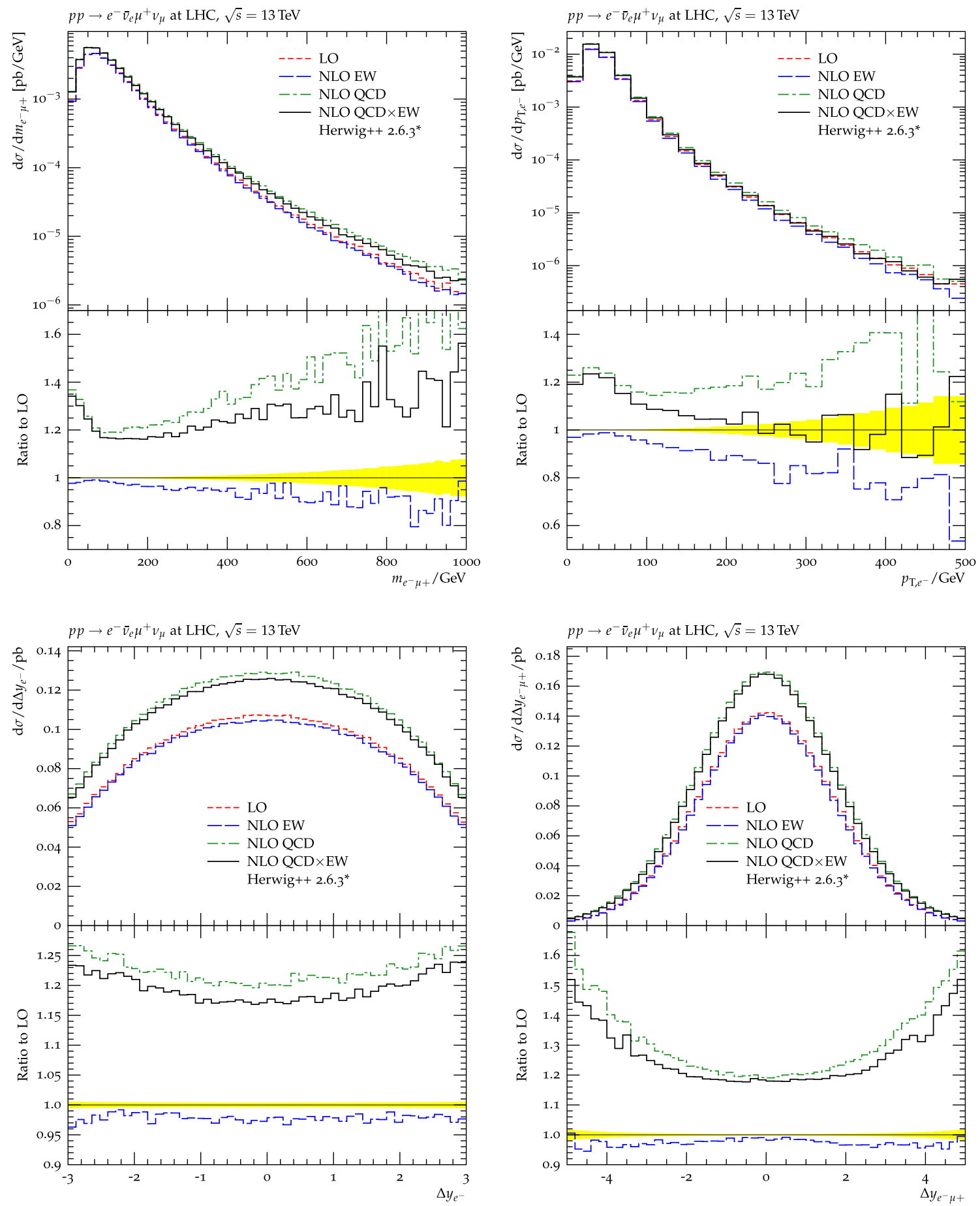

Fig. 16 Results from WW events at $\sqrt{s}=13 \mathrm{TeV}$. We show observables that can be reconstructed from the visible leptonic final state after we applied the veto (4.4)

QCD corrections introduced in this paper essentially requires a "back-to-back" signature of the vector bosons in the final state. Otherwise, events at high transverse momenta are dominated by hard QCD radiation, and the center-of-mass frame of the two vector bosons is strongly boosted in the transverse direction. In this kinematic regime, however, a naive factorization of EW and QCD corrections will almost surely fail, and substantial uncertainties due to missing terms of $\mathcal{O}\left(\alpha \alpha_{s}\right)$ (and also $\mathcal{O}\left(\alpha_{s}^{2}\right)$ ) will arise. Therefore, we strongly recommend the implementation of the veto (4.4) (or any equivalent 
prescription) for the application of the $K$-factor approach described in this work. ${ }^{3}$

Having established the role of the veto on the leptonic final state, we present results of differential distributions in leptonic observables. We apply the same selection of final states as described above in Sects. 2.3 and 4. Now, the leptons are, however, selected from a full hadronic final state with an additional isolation cut of $R=0.2$. Furthermore, the final state is of course modified by parton showers and hadronization as well as additional soft and collinear photon radiation. We left the underlying event switched off as we expect only a small effect for the observables presented here. In all cases we have generated 10M unvetoed events. The leptonic veto has only been applied at the analysis level.

In Fig. 14 we show a number of observables for the final state of $\mathrm{ZZ}$ production. In all cases we show four lines: the leading-order result (LO), results with only electroweak (NLO EW) or QCD corrections (NLO QCD) applied and, finally, the result with the combined EW and QCD corrections in the multiplicative scheme as outlined above. In both cases, with and without NLO QCD corrections, the additional EW correction is as sizable as in the partonic case, i.e. of the order of $-5 \%$ for small $m_{4 l}$, reaching up to $-20 \%$ for $m_{4 l}$ close to $1 \mathrm{TeV}$ or $p_{\mathrm{T}, l \bar{l}}$ close to $500 \mathrm{GeV}$. The rapidity distributions, shown in the lower two plots of Fig. 14, receive the correction of $-4 \%$ typical for the low- $\hat{s}$ configuration.

A similar picture emerges in the case of $\mathrm{WZ}$ production which we show in Fig. 15 for $\sqrt{s}=13 \mathrm{TeV}$. Here, the EW corrections are smaller than in the $\mathrm{ZZ}$ case. In every observable we find that the EW corrections act quite similar on the final states with and without NLO QCD corrections. While the QCD corrections are moderate, at the level of $20 \%$, the EW corrections vary between zero and $-20 \%$ and are again sizable for high transverse momenta of the $\mathrm{Z}$ bosons.

Finally, in Fig. 16 we consider selected observables for the case of W-pair production at $13 \mathrm{TeV}$. Here, the EW corrections are slightly larger than in the WZ case, but the overall picture remains the same. The QCD corrections are quite large but tamed by our veto on the leptonic final state, typically of the order of $20 \%$. The EW corrections are typically of the order of $5 \%$ but again sizable in the case of large lepton transverse momentum, where they can completely compensate the QCD corrections.

\section{Summary and conclusions}

We have computed the full NLO EW corrections to resonant vector-boson pair production at the LHC, taking into

\footnotetext{
${ }^{3}$ Furthermore, a "back-to-back" requirement for the final-state vector bosons will also reduce the admixture of massive-vector-boson radiation and $V_{1} V_{2} \gamma$ events, as well as contributions from quark-photon-induced processes, which suffer substantially from the large photon-PDF error quoted by the NNPDF collaboration.
}

account leptonic decays and corresponding spin correlations. We propose a simple and straightforward method-relying on unpolarized $2 \rightarrow 2 \mathrm{~K}$-factors-to implement our results in any state-of-the-art MC generator.

The EW corrections are combined with precise QCD predictions in a multiplicative approach, which is assumed to provide reliable results in a leading-order-like kinematic regime. We enforce this by application of a kinematic veto based on the transverse momenta of the leptonic decay products. To estimate the effect of the veto on QCD uncertainties from missing higher orders, we vary the renormalization and factorization scale. A similar behavior is observed for inclusive and exclusive cross sections, suggesting uncertainties of the order of 10-20\%. The crossing of the error bands for $p_{\mathrm{T}, l \bar{l}}$ around $120 \mathrm{GeV}$, as observed in Fig. 12, should not be interpreted as vanishing theory uncertainty. It will be affected by a NNLO calculation and deserves further studies.

We emphasize that our method also allows for an $a$ posteriori implementation of EW $K$-factors into MC samples that have already been generated. To demonstrate the practicability of our approach, we have included our corrections in the HERWIG++ MC generator and presented various distributions for four-lepton production at the LHC obtained in the HERWIG++ setup, including EW corrections, NLO QCD corrections matched to parton showers, as well as hadronization effects. In the future our method could also be applied to other process classes, such as $V+$ jet production at the LHC, allowing for phenomenological studies that combine EW precision on the one hand and an adequate treatment of dominating QCD effects on the other.

Acknowledgments This work was supported by the DFG Sonderforschungsbereich/Transregio 9 "Computergestützte Theoretische Teilchenphysik". SG acknowledges support from the EU Initial Training Network "MCnetITN" and the Helmholtz Alliance "Physics at the Terascale".

Open Access This article is distributed under the terms of the Creative Commons Attribution License which permits any use, distribution, and reproduction in any medium, provided the original author(s) and the source are credited.

Funded by $\mathrm{SCOAP}^{3}$ / License Version CC BY 4.0.

\section{Appendix A: Endpoint contributions from dipoles}

According to Ref. [42] the endpoint contribution compensating soft and collinear QED singularities from the virtual corrections of the unpolarized $V$-boson pair-production process $q \bar{q}^{\prime} \rightarrow V_{1} V_{2}$ is given by the general expression

$$
\begin{aligned}
\mathrm{d} \sigma_{q \bar{q}^{\prime} \rightarrow V_{1} V_{2} \gamma}^{\mathrm{E}}= & -\frac{\alpha}{2 \pi} \sum_{I \neq J} Q_{I} \sigma_{I} Q_{J} \sigma_{J} G_{I J}^{(\mathrm{sub})}\left(P_{I J}, m_{I}, m_{J}\right) \\
& \times \mathrm{d} \sigma_{q \bar{q}^{\prime} \rightarrow V_{1} V_{2}}^{\mathrm{LO}}\left(p_{I}, p_{J}\right),
\end{aligned}
$$


where the sum runs over all dipole contributions $(I J)$ of charged external particles, i.e. $I, J=q, \bar{q}^{\prime}, \mathrm{W}^{ \pm}$, and the universal (i.e. process-independent) functions $G_{I J}^{(\text {sub) }}$ $\left(P_{I J}, m_{I}, m_{J}\right)$ carry the respective endpoint singularities. The charge flow of the external particle $J$ is denoted by $\sigma_{J}$. Let us again state explicitly that the sum of the one-loop virtual corrections and the endpoint contribution, $\sigma_{q \bar{q}^{\prime} \rightarrow V_{1} V_{2}}^{\mathrm{V}}+$ $\sigma_{q \bar{q}^{\prime} \rightarrow V_{1} V_{2} \gamma}^{\mathrm{E}}$, is IR finite by construction and defined on a LO phase space solely parametrized by $\hat{s}$ and $\hat{t}$. In our computation the quark masses are neglected whenever possible and only introduced to regularize collinear singularities, whereas a finite $\mathrm{W}$ mass is kept throughout the calculation. Therefore, the dipole formulas for massive FS particles and massless IS particles have to be applied here.

In the case of a massless IS emitter $a$ and a massless IS spectator $b$ we find $P_{a b}=\sqrt{\hat{s}}$ and

$G_{a b}^{(\text {sub) }}\left(\hat{s}, m_{a}^{2}\right)=\mathcal{L}\left(\hat{s}, m_{a}^{2}\right)-\frac{\pi^{2}}{3}+2$,

with the auxiliary function

$$
\begin{aligned}
\mathcal{L}\left(P^{2}, m^{2}\right)= & \ln \left(\frac{m^{2}}{P^{2}}\right) \ln \left(\frac{\lambda^{2}}{P^{2}}\right)+\ln \left(\frac{\lambda^{2}}{P^{2}}\right) \\
& -\frac{1}{2} \ln ^{2}\left(\frac{m^{2}}{P^{2}}\right)+\frac{1}{2} \ln \left(\frac{m^{2}}{P^{2}}\right) .
\end{aligned}
$$

Here, soft singularities are regularized by an infinitesimal photon mass $\lambda$, and a small quark mass $m_{a}$ is kept to regularize collinear singularities, as mentioned before. For the case of a massive FS emitter and a massive FS spectator, which contributes to W-pair production, $G_{i j}^{\text {(sub) }}\left(P_{i j}^{2}, m_{i}, m_{j}\right)$ is given by Eq. (4.10) from Ref. [42], with $P_{i j}^{2}=\hat{s}$. The endpoint contributions $G_{a i}^{\text {(sub) }}\left(P_{i a}^{2}, m_{a}, m_{i}\right)$ and $G_{i a}^{(\mathrm{sub})}\left(P_{i a}^{2}, m_{i}\right)$ for massless IS emitters (spectators) and massive FS spectators (emitters) can be found in Eq. (7.4) of the respective paper, where $m_{a}$ has been set to zero in the expression for $G_{i a}^{\text {(sub) }}\left(P_{i a}^{2}, m_{i}\right)$. Here, the auxiliary momentum is given by $P_{i a}=p_{i}-p_{a}$.

Note that the finite terms (i.e. terms not proportional $\ln (\lambda)$ or $\ln \left(m_{q}\right)$ ) of the endpoint contributions in (A.1) do not have any physical meaning, since they are tailored to cancel certain subtraction contributions from the real-radiation processes, which are neglected anyway in the $\mathrm{V}+\mathrm{E}$ approximation. Nevertheless, our approach is still justified since in the $\mathrm{V}+\mathrm{E}$ approximation applied in this paper we do not claim to control the QED part of the EW corrections, and, more importantly, we have explicitly shown in Sect. 2.3 that this approximation works remarkably well in $V$-pair production processes. However, we clearly point out that this might be different for different process classes, and the validity of the $\mathrm{V}+\mathrm{E}$ approximation has to be carefully checked if applied to other processes.

\section{References}

1. G. Aad et al. [ATLAS Collaboration], JHEP 1303, 128 (2013) arXiv:1211.6096 [hep-ex]

2. G. Aad et al. [ATLAS Collaboration], Phys. Rev. D 87, 112001 (2013). arXiv:1210.2979 [hep-ex]

3. G. Aad et al. [ATLAS Collaboration], Eur. Phys. J. C 72, 2173 (2012). arXiv:1208.1390 [hep-ex]

4. S. Chatrchyan et al. [CMS Collaboration]. arXiv:1306.1126 [hep-ex]

5. S. Chatrchyan et al. [CMS Collaboration], Phys. Lett. B 721, 190 (2013). arXiv:1301.4698 [hep-ex]

6. S. Chatrchyan et al. [CMS Collaboration], JHEP 1301, 063 (2013). arXiv:1211.4890 [hep-ex]

7. A. Bierweiler, T. Kasprzik, H. Kühn, S. Uccirati, JHEP 1211, 093 (2012). arXiv:1208.3147 [hep-ph]

8. A. Bierweiler, T. Kasprzik, J.H. Kühn. arXiv:1305.5402 [hep-ph]

9. F. Caola, J.M. Henn, K. Melnikov, V.A. Smirnov. arXiv: 1404.5590 [hep-ph]

10. T. Gehrmann, L. Tancredi, E. Weihs, JHEP 1308, 070 (2013). arXiv:1306.6344 [hep-ph]

11. G. Chachamis, M. Czakon, D. Eiras, JHEP 0812, 003 (2008). arXiv:0802.4028 [hep-ph]

12. Z. Bern, A. De Freitas, L.J. Dixon, JHEP 0109, 037 (2001). hep-ph/0109078

13. S. Catani, L. Cieri, D. de Florian, G. Ferrera, M. Grazzini, Phys. Rev. Lett. 108, 072001 (2012). arXiv:1110.2375 [hep-ph]

14. M. Grazzini, S. Kallweit, D. Rathlev, A. Torre. arXiv:1309.7000 [hep-ph]

15. F. Campanario, S. Sapeta, Phys. Lett. B 718, 100 (2012). arXiv:1209.4595 [hep-ph]

16. F. Campanario, M. Rauch, S. Sapeta. arXiv:1309.7293 [hep-ph]

17. S. Dawson, I.M. Lewis, M. Zeng. arXiv:1307.3249 [hep-ph]

18. F. Cascioli, T. Gehrmann, M. Grazzini, S. Kallweit, P. Maierhfer, A. von Manteuffel, S. Pozzorini, D. Rathlev et al. arXiv:1405.2219 [hep-ph]

19. E. Accomando, A. Kaiser, Phys. Rev. D 73, 093006 (2006). hep-ph/0511088

20. E. Accomando, A. Denner, A. Kaiser, Nucl. Phys. B 706, 325 (2005). hep-ph/0409247

21. M. Billoni, S. Dittmaier, B. Jäger, C. Speckner. arXiv:1310.1564 [hep-ph]

22. J.H. Kühn, F. Metzler, A.A. Penin, S. Uccirati, JHEP 1106, 143 (2011). arXiv:1101.2563 [hep-ph]

23. J. Baglio, L.D. Ninh, M.M. Weber. arXiv:1307.4331 [hep-ph]

24. G. Chachamis. arXiv:1312.4220 [hep-ph]

25. R. Boughezal, Y. Li, F. Petriello. arXiv:1312.3972 [hep-ph]

26. W.B. Kilgore, C. Sturm. arXiv:1107.4798 [hep-ph]

27. A. Kotikov, J.H. Kühn, O. Veretin, Nucl. Phys. B788, 47-62 (2008). hep-ph/0703013 [HEP-PH]

28. Q.H. Cao, C.P. Yuan, Phys. Rev. Lett. 93, 042001 (2004). hep-ph/0401026

29. B.F.L. Ward, C. Glosser, S. Jadach, S.A. Yost, Int. J. Mod. Phys. A 20, 3735 (2005). hep-ph/0411047

30. B.F.L. Ward, S.A. Yost, Acta Phys. Polon. B 38, 2395 (2007). arXiv:0704.0294 [hep-ph]

31. A. Vicini et al., PoS RADCOR2007 (2007) 013

32. G. Balossini, G. Montagna, C.M. Carloni Calame, M. Moretti, O. Nicrosini, F. Piccinini, M. Treccani, A. Vicini, JHEP 1001 (2010) 013. arXiv:0907.0276 [hep-ph]

33. G. Balossini et al., Nuovo Cim. 123B, 741 (2008)

34. S. Dittmaier, A. Huss, C. Schwinn. arXiv:1405.6897 [hep-ph]

35. M. Bähr, S. Gieseke, M.A. Gigg, D. Grellscheid, K. Hamilton, O. Latunde-Dada, S. Plätzer, P. Richardson et al., Eur. Phys. J. C 58, 639 (2008). arXiv:0803.0883 [hep-ph] 
36. K. Arnold, L. d'Errico, S. Gieseke, D. Grellscheid, K. Hamilton, A. Papaefstathiou, S. Plätzer, P. Richardson et al. arXiv:1205.4902 [hep-ph]

37. A.D. Martin et al., Eur. Phys. J. C63, 189-285 (2009). arXiv:0901.0002 [hep-ph]

38. M.R. Whalley, D. Bourilkov, R.C. Group, in HERA and the LHC, ed. by A. de Roeck, H. Jung (CERN-2005-014, Geneva, 2005), p. 575. hep-ph/0508110

39. A.D. Martin, R.G. Roberts, W.J. Stirling, R.S. Thorne, Eur. Phys. J. C39, 155-161 (2005). hep-ph/0411040

40. M. Roth, S. Weinzierl, Phys. Lett. B 590, 190 (2004). hep-ph/0403200

41. R.D. Ball et al. [The NNPDF Collaboration]. arXiv:1308.0598 [hep-ph]

42. S. Dittmaier, Nucl. Phys. B 565, 69 (2000). hep-ph/9904440

43. K. Hamilton, JHEP 1101, 009 (2011). arXiv:1009.5391 [hep-ph]

44. H.-L. Lai, M. Guzzi, J. Huston, Z. Li, P.M. Nadolsky, J. Pumplin, C.-P. Yuan, Phys. Rev. D 82, 074024 (2010). arXiv:1007.2241 [hep$\mathrm{ph}]$

45. A. Denner, S. Dittmaier, M. Roth, L.H. Wieders, Nucl. Phys. B 724, 247 (2005). hep-ph/0505042

46. A. Denner, S. Dittmaier, Nucl. Phys. Proc. Suppl. 160, 22 (2006). hep-ph/0605312

47. A. Denner, S. Dittmaier, M. Roth, D. Wackeroth, Nucl. Phys. B 587, 67 (2000). hep-ph/0006307

48. J. Küblbeck, M. Böhm, A. Denner, Comput. Phys. Commun. 60, 165 (1990)
49. H. Eck, J. Küblbeck, Guide to FeynArts 1.0, University of Würzburg (1992)

50. T. Hahn, Comput. Phys. Commun. 140, 418 (2001). hep-ph/0012260

51. T. Hahn, M. Pérez-Victoria, Comput. Phys. Commun. 118, 153 (1999). hep-ph/9807565

52. T. Hahn, C. Schappacher, Comput. Phys. Commun. 143, 54-68 (2002). hep-ph/0105349

53. G.J. van Oldenborgh, J.A.M. Vermaseren, Z. Phys. C46, 425-438 (1990)

54. J. Alwall et al., JHEP 0709, 028 (2007). arXiv:0706.2334 [hep-ph]

55. K. Hamilton, P. Richardson, JHEP 0607, 010 (2006). hep-ph/0603034

56. D.R. Yennie, S.C. Frautschi, H. Suura, Ann. Phys. 13, 379 (1961)

57. P. Falgari, A.S. Papanastasiou, A. Signer, JHEP 1305, 156 (2013). arXiv: 1303.5299 [hep-ph]

58. M. Rubin, G.P. Salam, S. Sapeta, JHEP 1009, 084 (2010). arXiv: 1006.2144 [hep-ph]

59. S. Frixione, P. Nason, C. Oleari, JHEP 0711, 070 (2007). arXiv:0709.2092 [hep-ph]

60. A. Buckley, J. Butterworth, S. Gieseke, D. Grellscheid, S. Höche, H. Hoeth, F. Krauss, L. Lönnblad et al., Phys. Rept. 504, 145 (2011). arXiv:1101.2599 [hep-ph]

61. S. Gieseke, Prog. Part. Nucl. Phys. 72, 155 (2013) 